\title{
Efficient reconstruction of dispersive dielectric profiles using time domain reflectometry (TDR)
}

\author{
P. Leidenberger ${ }^{1}$, B. Oswald ${ }^{1, *}$, and K. Roth $^{1}$ \\ ${ }^{1}$ Institute of Environmental Physics, University of Heidelberg, Heidelberg, Germany \\ *now at: Paul Scherrer Institute, Villigen, Switzerland
}

Received: 29 July 2005 - Published in Hydrol. Earth Syst. Sci. Discuss.: 15 August 2005

Revised: 20 January 2006 - Accepted: 10 February 2006 - Published: 11 April 2006

\begin{abstract}
We present a numerical model for time domain reflectometry (TDR) signal propagation in dispersive dielectric materials. The numerical probe model is terminated with a parallel circuit, consisting of an ohmic resistor and an ideal capacitance. We derive analytical approximations for the capacitance, the inductance and the conductance of three-wire probes. We couple the time domain model with global optimization in order to reconstruct water content profiles from TDR traces. For efficiently solving the inverse problem we use genetic algorithms combined with a hierarchical parameterization. We investigate the performance of the method by reconstructing synthetically generated profiles. The algorithm is then applied to retrieve dielectric profiles from TDR traces measured in the field. We succeed in reconstructing dielectric and ohmic profiles where conventional methods, based on travel time extraction, fail.
\end{abstract}

\section{Introduction}

\subsection{Motivation}

Time Domain Reflectometry (TDR) has become an indispensable technique for measuring the water content of soils in hydrology, civil engineering, agriculture and related fields over the last years, for a review see Robinson et al. (2003). Early realizations of the method delivered a single water content $\theta$ from a TDR trace (Birchak et al., 1974; Topp et al., 1980, 1982a,b; Topp and Davis, 1985; Dasberg and Dalton, 1985). A second phase of TDR development has targeted to deliver spatially resolved water content profiles along the TDR probe (Yanuka et al., 1988; Hook et al., 1992; Dasberg and Hopmans, 1992; Lundstedt and Ström, 1996; Norgren and He, 1996; Pereira, 1997; Todoroff et al., 1998; Feng

Correspondence to: B. Oswald

(benedikt.oswald@psi.ch) et al., 1999; Oswald, 2000; Oswald et al., 2003; Lin, 2003; Heimovaara et al., 2004; Schlaeger, 2005).

Because the dielectric permittivity of soil material typically depends considerably on frequency, particularly if there are clay and loam components (Hoekstra and Delaney, 1974; Sposito and Prost, 1982; Ishida et al., 2000; Huisman et al., 2004; Robinson et al., 2005), in a third phase methods have been studied to recover the average dispersive dielectric parameters from TDR traces (Heimovaara, 1994; Heimovaara et al., 1996; Hilhorst et al., 2001; Lin, 2003). Clearly, the next logical step are methods to extract the full dielectric profile from a TDR trace.

\subsection{Objectives}

In this paper we study an efficient method for the reconstruction of spatially resolved profiles of water content and electrical conductivity from TDR traces assuming dispersive dielectric properties of the soil material along the probe. In particular, we want to reconstruct field measured TDR traces (Wollschläger and Roth, 2005) which could not be successfully reconstructed with techniques used by Roth et al. (1990).

We use the Debye model to account for dispersive dielectric properties (Debye, 1929). While the three-rod probe is often employed for TDR measurements, there is only scarce material on its transmission line parameters, particularly inductance, capacitance and conductance per unit length. We therefore derive an analytical model for these parameters under the approximation of small conductor diameter $D$ with respect to conductors' center distance $d$. We assess the accuracy of the analytical model by comparing its predictions to a full-wave numerical analysis, using an established, commercially available program.

Published by Copernicus GmbH on behalf of the European Geosciences Union. 


\section{Methods}

The propagation of TDR signals, voltage $v(x, t)$ and current $i(x, t)$, on probes of two or more conducting rods is described by transmission line theory (e.g. Ramo et al., 1984). Our approach for numerically modeling TDR probes is essentially based on Oswald et al. (2003). A transmission line is described by capacitance $C^{\prime}$, conductance $G^{\prime}$, inductance $L^{\prime}$ and resistance $R^{\prime}$, all per unit length, respectively. These parameters are functions of the probe geometry and the dielectric and ohmic properties of the material between the probe's conductors $C^{\prime}=C^{\prime}(d, D, \epsilon), G^{\prime}=G^{\prime}(d, D, \sigma)$, $L^{\prime}=L^{\prime}(d, D, \mu)$ and $R^{\prime}=R^{\prime}\left(d, D, R_{\text {skin }}\right)$ where $d$ is the spacing between the probe rods (for a three rod probe this is the distance between neighboring rods) and $D$ is the diameter of the probe rods. We assume rods of identical diameter.

For piecewise constant transmission line parameters, voltage $v(x, t)$ and current $i(x, t)$ are described by the the following two linear first order, partial differential equations (PDE) (Ramo et al., 1984):

$$
\begin{aligned}
& \frac{\partial v}{\partial x}=-\left(R^{\prime}+L^{\prime} \frac{\partial}{\partial t}\right) i \\
& \frac{\partial i}{\partial x}=-\left(G^{\prime}+C^{\prime} \frac{\partial}{\partial t}\right) v
\end{aligned}
$$

The piecewise constant dielectric permittivity $\epsilon$ and ohmic conductivity $\sigma$ can be discontinuous, because the water content $\theta$ in general is discontinuous across soil boundaries. With a variable water content $\theta(x)$ along the probe the parameters $G^{\prime}$ and $C^{\prime}$ vary accordingly; $L^{\prime}$ is assumed to be constant, because the materials' magnetic permeability equals $\mu_{0}$; resistance $R^{\prime}$, caused by skin effect, is neglected in the current study.

For extracting dielectric and ohmic profiles from measured TDR traces we use an iterative, globally optimizing approach based on Oswald et al. (2003) in order to solve the non-linear, inverse, electromagnetic problem. The global optimization method uses genetic algorithms (GA) from a publicly available library Levine (1996).

To calculate the TDR signal for a given dielectric profile we numerically solve Eqs. (1) and (2) using a finite difference time domain (FDTD) approach (Taflove, 1998). The spatial discretization of the $x$ coordinate is given by $x=k \Delta x$ and temporal discretization by $t=n \Delta t$ with:

$\Delta x \leq \frac{\lambda_{\min }}{10}$

where $\lambda_{\min }$ is the minimum wavelength present in the system, which in non-magnetic material, is determined by the maximum frequency $f_{\max }$ and the largest permittivity value $\epsilon_{\max }$ (Taflove, 1998):

$\lambda_{\min }=\frac{c_{0}}{f_{\max } \sqrt{\epsilon_{r, \max }}}$.
We estimate the maximum relevant frequency from

$t_{\text {rise }} \cdot f_{3 \mathrm{~dB}}=0.34$,

an expression widely used in electrical engineering. It refers to a Gaussian type time domain waveform with rise time $t_{\text {rise }}$. This is a good model for a TDR input signal.

To keep the explicit time domain integration scheme stable, an upper limit for $\Delta t$ must be observed (Taflove, 1998; Kunz and Luebbers, 1993):

$\Delta t \leq \frac{\Delta x}{c_{0}}$

\subsection{Numerical solution of transmission line equations}

Numerically, there are three spatially different regions, at the beginning of the probe $x=0$, at the end of the probe, $x=\Lambda$, and in-between, $x<0<\Lambda$. At the ends of the probe, the discretized set of PDE is connected to a lumped electrical model, such as voltage sources or resistive-capacitive terminations.

\subsubsection{Boundary conditions}

The termination of a TDR probe is modeled with a parallel circuit, consisting of an ohmic resistor and an ideal capacitance. The voltage current relationship of this parallel circuit is given by

$I_{T}=\frac{V_{T}}{R_{T}}+C_{T} \frac{\partial V_{T}}{\partial t}$

where $I_{T}$ is the current at the end of the TDR probe through the terminal resistor $R_{T}$ and the terminal capacitance $C_{T}$. $V_{T}$ is the voltage drop at the end of the TDR probe over the parallel circuit of $R_{T}$ and $C_{T}$. To couple this parallel circuit to the distributed transmission line model we use Eq. (1). We truncate the FDTD scheme of the probe through coupling Eqs. (1) and (7) using the definitions:

$$
\begin{aligned}
& i(x=\Lambda, t)=I_{T} \\
& v(x=\Lambda, t)=V_{T} .
\end{aligned}
$$

We rewrite Eq. (1)

$$
\begin{aligned}
& \frac{\partial v}{\partial x}=-R_{k}^{\prime} i-\left.L_{k}^{\prime} \frac{\partial i}{\partial t}\right|_{x=\Lambda} \\
& \Rightarrow \frac{\partial}{\partial x} v(x=\Lambda, t)=-R_{K}^{\prime} i(\Lambda, t)-L_{K}^{\prime} \frac{\partial}{\partial t} i(\Lambda, t) .
\end{aligned}
$$

All current terms in Eq. (11) are replaced by inserting Eq. (7). Note that the currents in the expressions, both constitutive and first-order PDE, are equivalent. Also, the voltages at the end of the probe and across the resistor are equal:

$$
\begin{aligned}
\frac{\partial}{\partial x} v(\Lambda, t)= & -\frac{R_{K}^{\prime}}{R_{T}} v(\Lambda, t)-R_{K}^{\prime} C_{T} \frac{\partial}{\partial t} v(\Lambda, t) \\
& -\frac{L_{K}^{\prime}}{R_{T}} \frac{\partial}{\partial t} v(\Lambda, t)-L_{K}^{\prime} C_{T} \frac{\partial^{2}}{\partial t^{2}} v(\Lambda, t) .
\end{aligned}
$$


We select a suitable discretization of Eq. (12): (i) the discretization must result in a fully explicit update scheme; (ii) the scheme must not require values outside the spatial computational domain $x=[0 \ldots \Lambda]$. We choose the "backward differencing in space" and "forward differencing in time" scheme using the Taylor series expansion of first-order accuracy. The sum of backward and forward second-order Taylor series expansion in time provides the second order time derivative. With the usual notation we write the discretized version of Eq. (12):

$$
\begin{aligned}
\left(\frac{v_{K}^{n}-v_{K-1}^{n}}{\Delta x}\right)= & -\frac{R_{K}^{\prime}}{R_{T}} v_{K}^{n}-R_{K}^{\prime} C_{T}\left(\frac{v_{K}^{n+1}-v_{K}^{n}}{\Delta t}\right) \\
& -\frac{L_{K}^{\prime}}{R_{T}}\left(\frac{v_{K}^{n+1}-v_{K}^{n}}{\Delta t}\right) \\
& -L_{K}^{\prime} C_{T}\left(\frac{v_{K}^{n+1}-2 v_{K}^{n}+v_{K}^{n-1}}{\Delta t^{2}}\right) .
\end{aligned}
$$

Finally, by rearranging Eq. (13) we obtain the explicit update procedure, in the time domain, for the voltage at the end of the TDR probe $x=\Lambda$.

$$
\begin{aligned}
v_{K}^{n+1}= & \left(\frac{R_{K}^{\prime} C_{T}}{\Delta t}+\frac{L_{K}^{\prime}}{R_{T} \Delta t}+\frac{L_{K}^{\prime} C_{T}}{\Delta t^{2}}\right)^{-1} \\
& \cdot\left[v _ { K } ^ { n } \left(\frac{2 L_{K}^{\prime} C_{T}}{\Delta t^{2}}+\frac{L_{K}^{\prime}}{R_{T} \Delta t}+\frac{R_{K}^{\prime} C_{T}}{\Delta t}-\frac{R_{K}^{\prime}}{R_{T}}\right.\right. \\
& \left.\left.-\frac{1}{\Delta x}\right)-\frac{L_{K}^{\prime} C_{T}}{\Delta t^{2}} v_{K}^{n-1}+\frac{1}{\Delta x} v_{K-1}^{n}\right]
\end{aligned}
$$

and similarly for the current at $x=\Lambda$ from, using Eq. (7):

$i_{K}^{n+1}=\frac{1}{R_{T}} v_{K}^{n+1}+C_{T} \frac{v_{K}^{n+1}-v_{K}^{n}}{\Delta t}$.

As special cases we mention $C_{T}=0, R_{T}<\infty$ and $C_{T}=0$, $R_{T} \rightarrow \infty$. An overview of the equations of all these boundary conditions is given in Table 1. We have implemented them in our TDR code, so almost any given experimental setup can be modeled. The values of $C_{T}$ and $R_{T}$ can also be optimized for, if so desired.

To implement the excitation we employ the same approach used by Oswald et al. (2003). We couple a resistive voltage source to the distributed transmission line. The resistive voltage source consists of a series of an ideal ohmic resistor $R_{S}$ and an ideal voltage source $v_{S}^{n}$. To avoid reflections between the voltage source and the cable connecting the TDR instrument to the probe we adjust $R_{S}$ to the impedance of the connecting cable. The current flow out of the resistive voltage source is $i_{S}^{n}$. The time derivative of the source voltage is implemented with a discretized version of the given expression for the time domain signal shape.

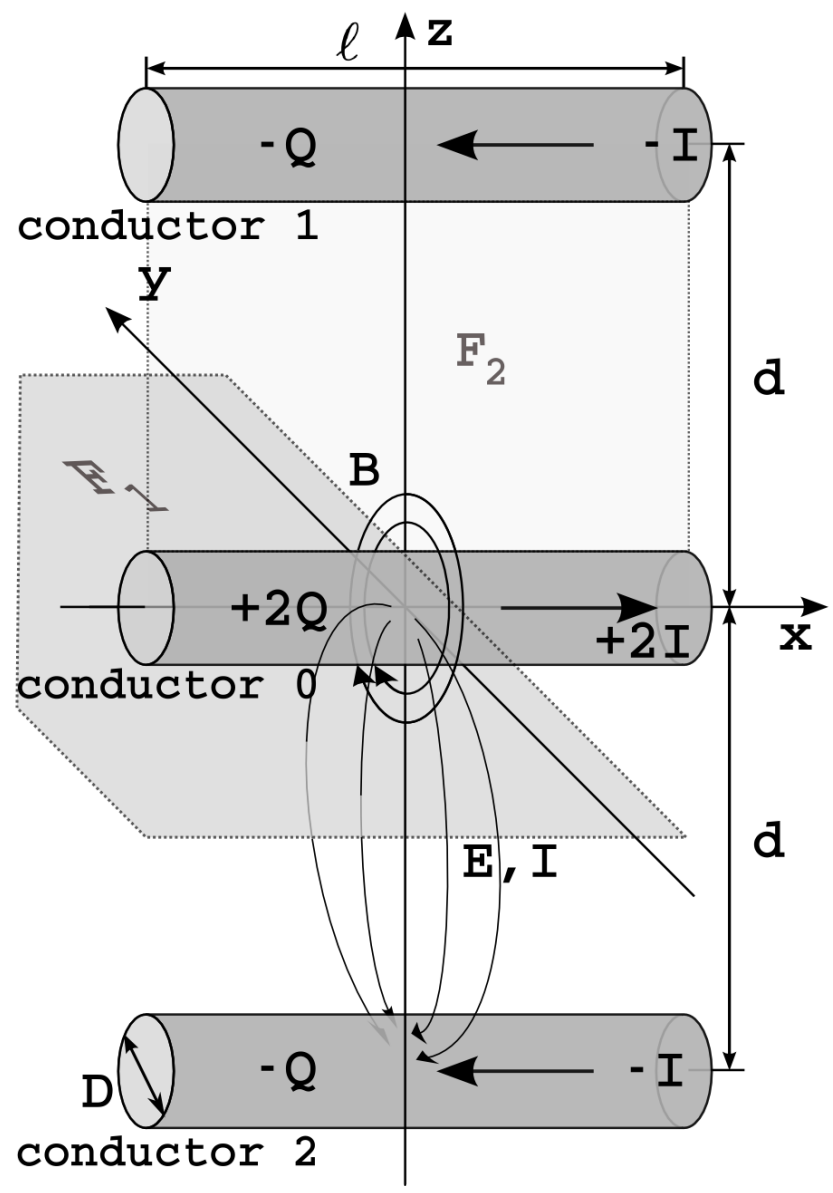

Fig. 1. Three-rod probe configuration and parameters.

\subsubsection{Transmission line parameters for three-rod TDR probe}

To solve the forward TDR problem the transmission line parameters for the three-rod probe, $C^{\prime}, G^{\prime}, L^{\prime}$ and $R^{\prime}$, are essential. Closed-form, analytical expressions for the tworod probe and the coaxial line are well known (Ramo et al., 1984). This is however not the case for the three-rod probe. We will derive an analytical model for the three-rod TDR probe based on an approximation of the electric and magnetic fields. The resulting transmission line parameters are compared to numerical simulations.

We calculate the electric parameters, $C^{\prime}$ and $G^{\prime}$, from the electric potential $\Phi_{\mathrm{el}}$ and the inductance $L^{\prime}$ from the magnetic induction $\boldsymbol{B}$ of the three-rod probe. For long rods and a large conductor distance $d$ in comparison to the conductor diameter $D$, i.e. $\frac{D}{d} \ll 1$, we approximate the electric potential and the magnetic induction. We postulate that the total electrostatic potential of a three-rod probe equals the superposition of the single conductor potentials; the same assumption applies for the magnetic induction. Thus, the neighboring conductors are neglected for the derivation of the potential of 
Table 1. Summary of boundary conditions.

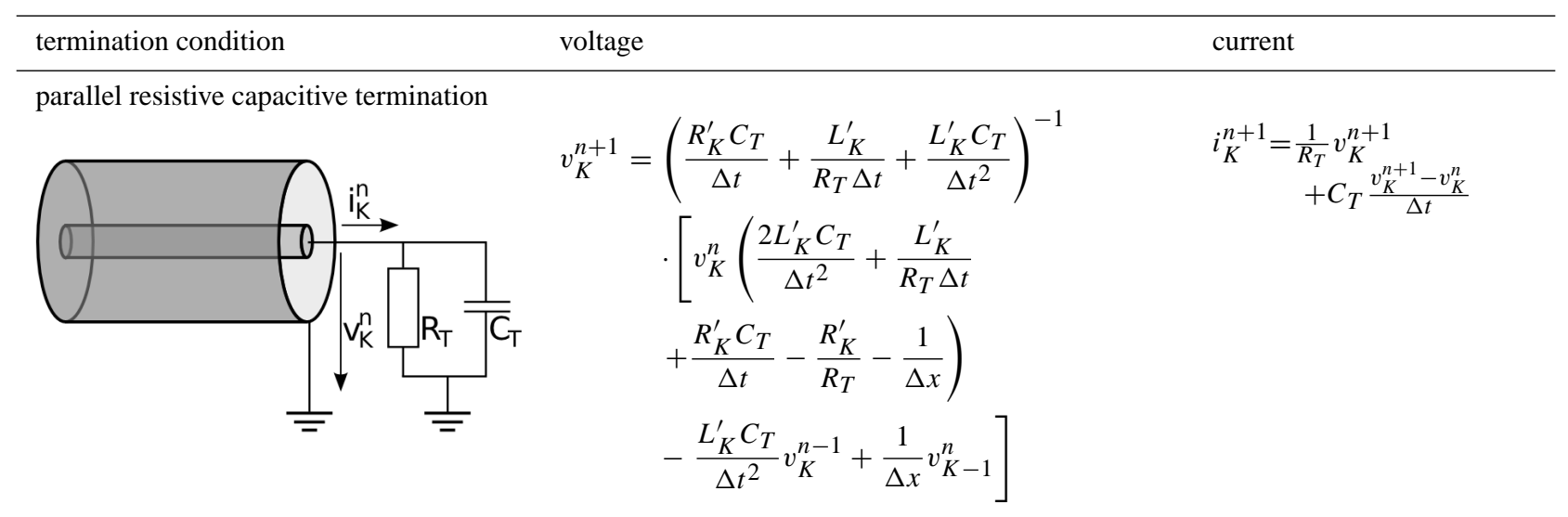

$$
\underbrace{\text { resistive termination }}_{(}
$$

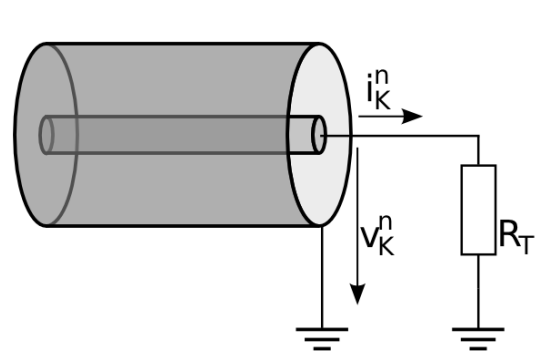

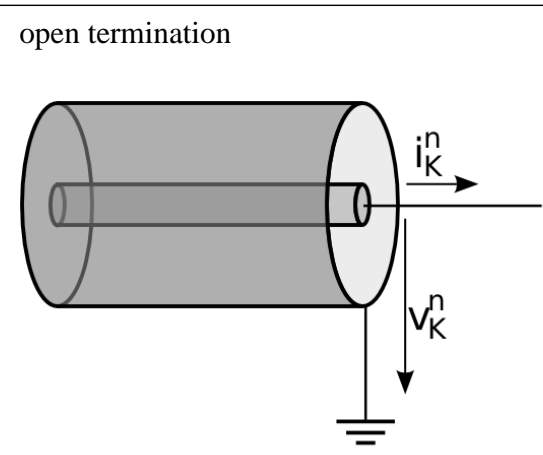

$$
v_{K}^{n+1}=v_{K-1}^{n+1}
$$$$
i_{K}^{n+1}=0
$$

$$
\begin{gathered}
\text { resistive voltage source termination } \\
+\frac{\Delta t R_{S}}{L_{1}^{\prime} \Delta x} v_{k+1}^{n+1}=\frac{\Delta t R_{1}^{\prime}}{L_{1}^{\prime}} v_{S}^{n} \\
+\Delta t \frac{\partial v_{S}^{n}}{\partial t}
\end{gathered}
$$

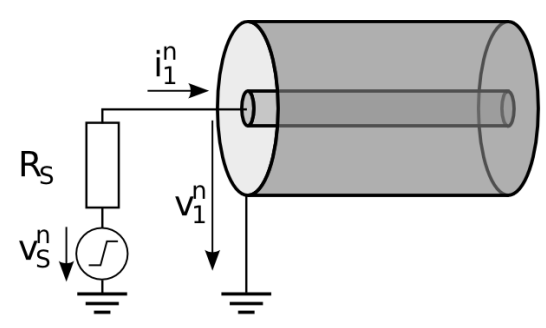

a specific conductor. The details of the derivation are given in Appendix A. The electrostatic potential, magnetic field, and the geometrical basis of the three-rod probe for calculat- ing these parameters are shown in Fig. 1. The transmission line parameters per unit length for the three rod probe with 
Table 2. Fit functions and parameters for numerical extracted transmission line parameters for a three rod probe. The fit functions following the structure of Eqs. (16)-(18). Validated for $\kappa=1.5 . .40$.

\begin{tabular}{|c|c|c|c|c|c|c|c|c|}
\hline & fit function & $a$ & $b$ & $c$ & $d$ & $e$ & $f$ & $g$ \\
\hline$C(\kappa)=$ & $\frac{a \pi \epsilon}{b+\ln (c \kappa+d)+e \ln (f \kappa+g)}$ & \multirow{2}{*}{$9.758 \cdot 10^{+1}$} & \multirow{2}{*}{1.090} & \multirow{2}{*}{$9.486 \cdot 10^{+1}$} & \multirow{2}{*}{$-1.421 \cdot 10^{+2}$} & \multirow{2}{*}{$7.516 \cdot 10^{+1}$} & \multirow{2}{*}{1.278} & \multirow{2}{*}{$3.772 \cdot 10^{-1}$} \\
\hline$G(\kappa)=$ & $\frac{a \pi \sigma}{b+\ln (c \kappa+d)+e \ln (f \kappa+g)}$ & & & & & & & \\
\hline$L(\kappa)=$ & $\begin{array}{l}\frac{a \mu}{\pi}(b+\ln (c \kappa+d) \\
+e \ln (f \kappa+g))\end{array}$ & $7.692 \cdot 10^{-3}$ & $7.857 \cdot 10^{-1}$ & $7.654 \cdot 10^{+1}$ & $-1.147 \cdot 10^{+2}$ & $9.846 \cdot 10^{+1}$ & 1.395 & $2.592 \cdot 10^{-1}$ \\
\hline
\end{tabular}
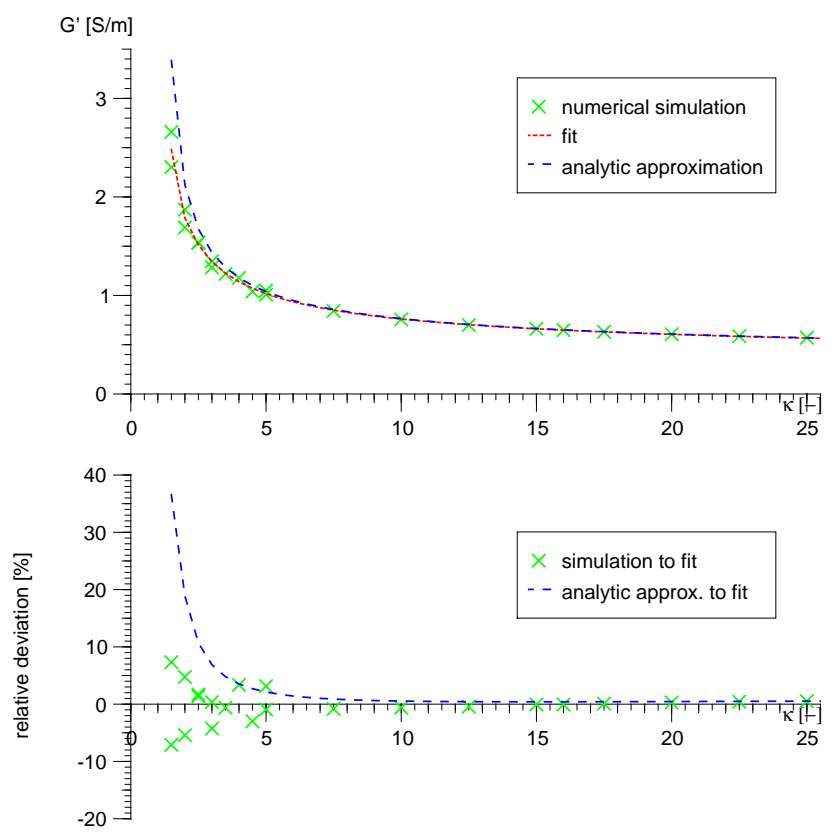

Fig. 2. Comparison of numerical and analytical extracted conductance per unit length $G^{\prime}$ for a three-rod probe as a function of probe geometry $\kappa=\frac{d}{D} ; \sigma=0.5$. Top: transmission line parameter from: (i) numerical simulations and a fit through them, Table 2; (ii) analytical approximation, Eq. (17). Bottom: relative deviation of the: (i) numerical results to the fit; (ii) approximative model for $G^{\prime}$ to the fit.

$\kappa=\frac{d}{D}$ are then obtained as

$$
\begin{aligned}
C^{\prime} & =\frac{4 \pi \epsilon}{\ln \left(\frac{4 \kappa^{2}-1}{4 \kappa-1}\right)+2 \ln (2 \kappa-1)} \\
G^{\prime} & =\frac{4 \pi \sigma}{\ln \left(\frac{4 \kappa^{2}-1}{4 \kappa-1}\right)+2 \ln (2 \kappa-1)} \\
L^{\prime} & =\frac{3 \mu_{0}}{4 \pi}\left[\ln (2 \kappa-1)+\frac{1}{3} \ln \left(\frac{2 \kappa+1}{4 \kappa-1}\right)\right] .
\end{aligned}
$$

For assessing the quality of the approximate solutions we use the commercially available 3-dimensional electrodynamics
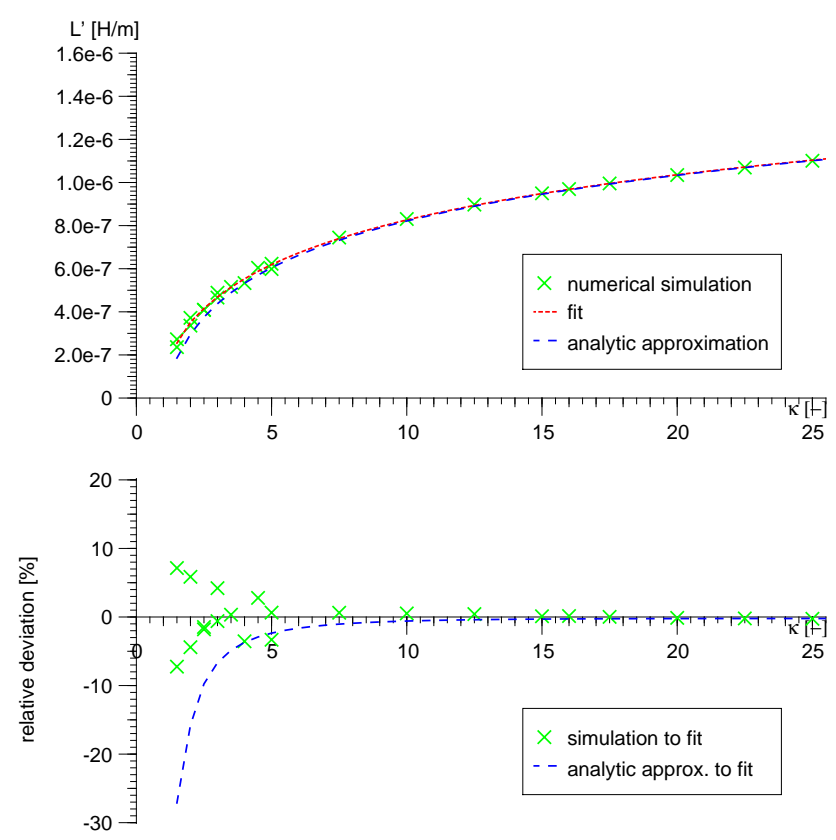

Fig. 3. Comparison of numerical and analytical extracted inductance per unit length $L^{\prime}$ for a three-rod probe as a function of probe geometry $\kappa=\frac{d}{D}$. Top: transmission line parameter from: (i) numerical simulations and a fit through them, Table 2; (ii) analytical approximation, Eq. (18); $\mu_{r}=1$. Bottom: relative deviation of the: (i) numerical results to the fit; (ii) approximative model for $L^{\prime}$ to the fit.

solver HFSS ${ }^{\mathrm{TM}}$ from Ansoft Inc. First, we determined the transmission line parameters for a two rod probe. The differences between the numerical values and the exact solution are less than $1 \%$. We then numerically evaluate the transmission line parameters of a three-rod probe using HFSS and fit them to an appropriately parameterized function. The results of the analytical model and the numerical simulations are shown in Figs. 2-3. Table 2 presents the used fit functions and their corresponding parameters.

Generally, the accuracy of results obtained from finite element analysis strongly depends on mesh size (Volakis et al., 1984): the smaller the tetrahedral mesh elements are, the better is the precision of results. At the same time, memory and 
computation time increase considerably. For $\kappa>5$ we get an excellent agreement between the analytic approximation and the heuristic fit basing on the numerical simulation. For $\kappa \leq 5$ the analytic approximation becomes inaccurate and the numeric simulation starts to oscillate. This is a problem of the discretization and can be alleviated by using a machine with more memory.

Calculating the propagation velocity with the fit functions for $L^{\prime}$ and $C^{\prime}$, via $c=\frac{1}{\sqrt{L^{\prime} C^{\prime}}}$, the maximum relative deviation of the speed of light $c_{0}$ is $0.14 \%$ over $\kappa=1.5$...25. For the two three rod TDR probes we use $\kappa$ is 4.69 and 6.25 so we get an error in speed of light $c_{0}$ of $0.12 \%$ and $0.13 \%$. The quality of the analytical model improves with increasing $\kappa$, corresponding more and more to the situation of infinitely thin line charges and current filaments, respectively.

\subsection{Time domain dispersive dielectric modeling}

Experience gained from TDR traces measured in the field has shown that it is mandatory to consider dispersive dielectric soil properties. We start with a Debye model using one single relaxation frequency (Debye, 1929; Nyfors and Vainikainen, 1989; Taflove, 1998). The Debye model describes the orientation polarization of polar molecules. Let us think of an electric field, switched on instantaneously. The polar molecules turn and the polarization evolves exponentially, with a time constant $\tau$, to its final state. The relative dielectric permittivity $\epsilon_{r}$ as a function of frequency is then:

$\epsilon_{r}(\omega)=\epsilon_{\infty}^{\prime}+\frac{\epsilon_{s}^{\prime}-\epsilon_{\infty}^{\prime}}{1+j \omega \tau}$.

Here $\epsilon_{\infty}^{\prime}$ is the permittivity at infinite frequency, where the orientation polarization of the molecules has no time to develop. The static permittivity $\epsilon_{s}^{\prime}$ corresponds to a state where the orientation polarization has had sufficient time to develop fully. For solving the transmission line equations in the time domain, we transform Eq. (19) into the time domain.

$\epsilon_{r}(t)=\epsilon_{\infty}^{\prime} \delta(t)+\frac{\Delta \epsilon^{\prime}}{\tau} e^{-\frac{t}{\tau}} U(t)$

with $\Delta \epsilon_{r}^{\prime}=\epsilon_{s}^{\prime}-\epsilon_{\infty}^{\prime}$. We end up with a time-dependent capacitance per unit length $C^{\prime}(t)$, which is split into a timedependent and a time-independent part:

$C^{\prime}(t)=C_{0}^{\prime} \epsilon_{r}(t)$

Equation (21) with Eqs. (2) and (1) are discretized, using central finite differences both in space and in time. We obtain the update procedure for the voltage and current:

$$
\begin{aligned}
v_{k}^{n+1}= & -\frac{2 \Delta t G_{k}^{\prime}}{C_{0 k}^{\prime} \epsilon_{\infty k}^{\prime}} v_{k}^{n}-\frac{2 \Delta t \Delta \epsilon_{k}^{\prime}}{\epsilon_{\infty k}^{\prime} \tau_{k}} v_{k}^{n}+v_{k}^{n-1} \\
& -\frac{\Delta t}{C_{0 k}^{\prime} \epsilon_{\infty k}^{\prime} \Delta x}\left(i_{k+1}^{n}-i_{k-1}^{n}\right)+\frac{2 \Delta \epsilon_{k}^{\prime} \Delta t}{\tau_{k}^{2} \epsilon_{\infty k}^{\prime}} \psi_{k}^{n}
\end{aligned}
$$

$i_{k}^{n+1}=-\frac{2 R_{k}^{\prime} \Delta t}{L_{k}^{\prime}} i_{k}^{n}+i_{k}^{n-1}-\frac{\Delta t}{\Delta x L_{k}^{\prime}}\left(v_{k+1}^{n}-v_{k-1}^{n}\right)$

with the abbreviation

$\psi_{k}^{n}=e^{-\frac{\Delta t}{\tau_{k}}} \psi_{k}^{n-1}+\frac{\Delta t}{2}\left(v_{k}^{n}+e^{-\frac{\Delta t}{\tau_{k}}} v_{k}^{n-1}\right)$.

The detailed calculation for this discretization can be found in Appendix B.

\subsection{Hierarchical optimization}

Our profile reconstruction approach is based on Oswald et al. (2003). The non-linear inverse problem is solved iteratively with a transmission line solver to calculate TDR traces, based on a given profile of electric parameters. The forward solver is embedded into a global optimizer based on a genetic algorithm (Levine, 1996; Rahmat-Samii and Michielssen, 1999) which delivers electric parameter profiles, adapted according to their fitness. Fitness is a quantity which is roughly inversely proportional to the trace mismatch:

$m=\sum_{n=N_{\text {start }}}^{N_{\text {stop }}}\left|v_{\text {meas }}(n \Delta t)-v_{\text {calc }}(n \Delta t)\right|$,

We use the sum of absolute values of the difference between calculated and measured TDR traces in contrast to the sum of squared differences (Oswald et al., 2003) because it is a robust estimator, typically used for non-Gaussian errors. In contrast the sum of squared differences, the $L^{2}$-norm, is only applicable to Gaussian noise. The genetic algorithm operates on bit-strings which are mapped to real numbers to produce the electric parameter profiles. Hence the electric parameters are inherently discretized. Using a sufficient number of bits per parameter we provide a fine-grained set of values. The efficiency of profile reconstruction depends on the genetic algorithm's parameters: mutation rate, crossover probability and population size. The corresponding values are listed in Tables 4 and 6.

While Oswald et al. (2003) achieve to solve the problem, there are still issues, namely (i) it is computationally intensive due to a large number of forward problem runs and (ii) the resulting electric parameter profiles may exhibit oscillatory behavior even if their average corresponds to the converged state.

To reduce the computational burden and to achieve smoother parameter profiles we have implemented a hierarchical optimization scheme (Fig. 4). The scheme starts out with a coarse spatial resolution which is increased as convergence rate decreases. For assessing the degree of convergence we calculate the envelope of the fitness and approximate its slope with a line (Fig. 5). An envelope point (squares at green line) is retrieved as the maximum fitness value of $N$ consecutive individuals, in our case $N=30$. A complete envelope consists of $M$ such points. As soon as the next $N$ individuals have been calculated, the oldest envelope point is 


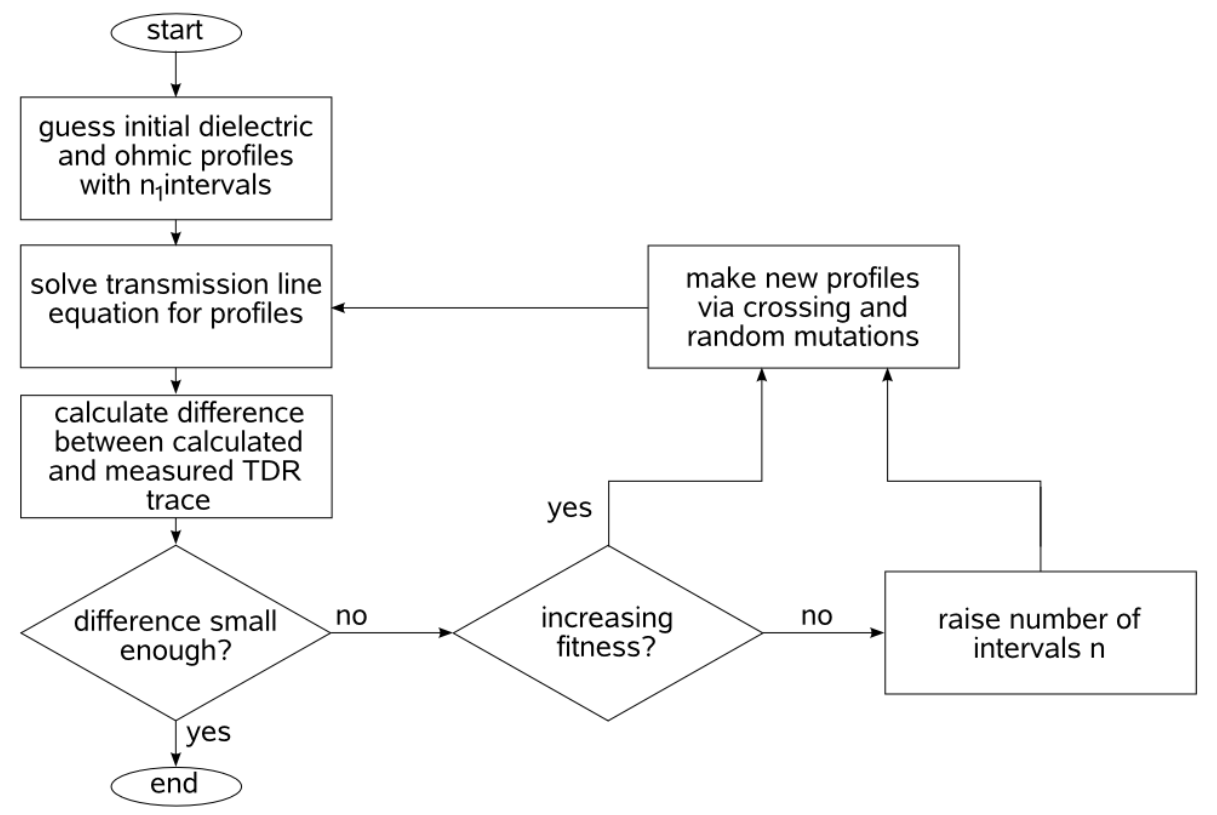

Fig. 4. Flowchart for the hierarchical optimization.

Table 3. Parameters used for calculation of synthetic TDR traces for validation and demonstration of termination conditions and the dispersive media, for all: probe length $=0.1 \mathrm{~m}$, TDR rise time $t_{\text {rise }}=300 \mathrm{ps}, \kappa=10$, conductor diameter $D=4 \mathrm{~mm}$.

\begin{tabular}{lcccccccc}
\hline Figure number & $\epsilon_{\infty}^{\prime}$ & $\Delta \epsilon^{\prime}$ & $f_{\text {rel }}(\mathrm{MHz})$ & $\sigma^{\prime}\left(\frac{\mathrm{s}}{\mathrm{m}}\right)$ & $R_{T}(\Omega)$ & $C_{T}(\mathrm{~F})$ & $t_{\mathrm{sec}}$ & $\Delta x(\mathrm{~m})$ \\
\hline 6 & 10 & - & - & $1 \cdot 10^{-30}$ & - & - & 0.25 & $1.2 \cdot 10^{-3}$ \\
7 & 1 & - & - & $1 \cdot 10^{-30}$ & 1438.8 & $5.0^{-1} \cdot 10^{-12}$ & 0.10 & $5.0 \cdot 10^{-4}$ \\
9 & 10 & 10 & 100 & $1 \cdot 10^{-30}$ & - & - & 0.25 & $1.2 \cdot 10^{-3}$ \\
8 (green line) & 10 & - & - & $1 \cdot 10^{-30}$ & 113 & - & 0.20 & $1.0 \cdot 10^{-3}$ \\
8 (red dotted line) & 10 & - & - & $1 \cdot 10^{-30}$ & 1436.8 & $5 \cdot 10^{-12}$ & 0.20 & $1.0 \cdot 10^{-3}$ \\
8 (blue dashed line) & 10 & - & - & $4 \cdot 10^{-2}$ & - & - & 0.20 & $1.0 \cdot 10^{-3}$ \\
10 (green line) & 10 & 10 & 10 & $1 \cdot 10^{-30}$ & - & - & 0.20 & $1.0 \cdot 10^{-3}$ \\
10 (red dotted line) & 10 & 5 & 100 & $1 \cdot 10^{-30}$ & - & - & 0.20 & $1.0 \cdot 10^{-3}$ \\
10 (blue dashed line) & 10 & 10 & 100 & $1 \cdot 10^{-30}$ & - & - & 0.20 & $1.0 \cdot 10^{-3}$ \\
\hline
\end{tabular}

discarded and the whole envelope section is moved one point ahead with respect to the sequence of evaluated individuals. If the majority of envelope points is below the line (red line), with the slope defined in the job file, the spatial resolution is increased by cutting the intervals of dielectric properties into halves. The new intervals are initialized with the same dielectric properties, as the old intervals had at the same location. The optimization stops if a previously specified spatial resolution is reached and the fitness does not increase.

\section{Results}

\subsection{Validation of parallel RC boundary condition}

We show the results of TDR traces calculated for different probe termination conditions with a non-dispersive dielectric permittivity between the probe conductors, for all parameters cf. Table 3. Figure 6 shows a comparison for the open termination, calculated with HFSS ${ }^{\mathrm{TM}}$ and our own code. All traces generated with HFSS ${ }^{\mathrm{TM}}$ were calculated under the assumptions (i) that the TDR signal source is connected directly to the probe and (ii) that the source has the same impedance as the probe. Therefore, there is no initial reflection.

The traces calculated with HFSS $^{\text {TM }}$ and our code are not totally identic. The small differences can not be neglected 


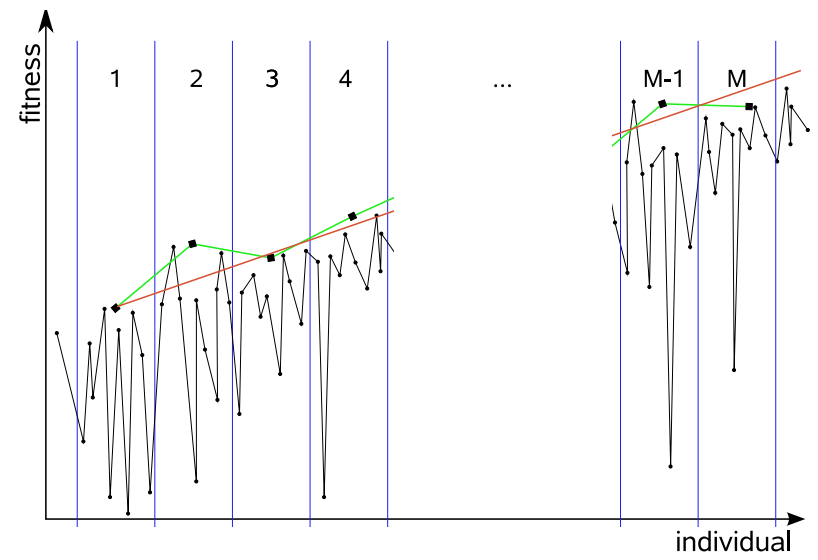

Fig. 5. Determination of the criteria when to increment spatial resolution.

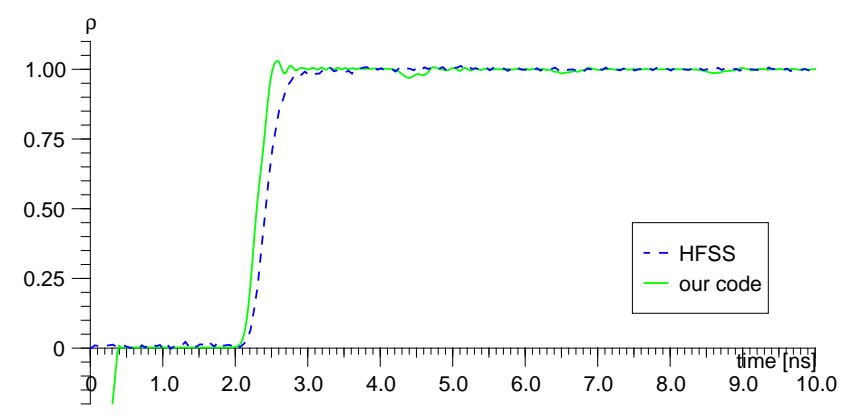

Fig. 6. Calculated TDR trace with HFSS (blue dashed line) and our code (green line) for two-rod probe with length $l=0.1 \mathrm{~m}, D=4 \mathrm{~mm}$, $\kappa=10, \epsilon_{\infty}^{\prime}=10, \sigma=10^{-30} \mathrm{~S} / \mathrm{m}$, not dispersive, infinite termination.

and are not a pure result of numeric simulation. We calculate the TDR probe fully 3-dimensional with $\mathrm{HFSS}^{\mathrm{TM}}$ so it includes the finite length of the transmission line. Our code uses the ideal transmission line equations, only valid for infinite long transmission lines. The influence of this effect is important in the content of spatial reconstruction of dielectric profiles. We expect a higher systematic error for the reconstructed dielectric parameters at the end of the probe, than for the rest.

Figure 7 shows the result of a probe with parallel resistive capacitive termination. At the reflection we can see the effect of the resistive capacitive termination: (i) in the beginning it behaves like a short circuit; (ii) if the capacitor is completely charged, it behaves like a pure resistive termination; (iii) and the edges of the reflections are smoothed. In Fig. 8 different termination conditions are calculated with our code. The TDR source here has an impedance of $50 \Omega$, therefore the first reflection results from the source-probe-transition, the second from the end of the probe. After these, there are multiple reflections. The TDR probe terminated with the probe's impedance produces the green trace. There are no reflections resulting from the end of the probe, as expected. The red

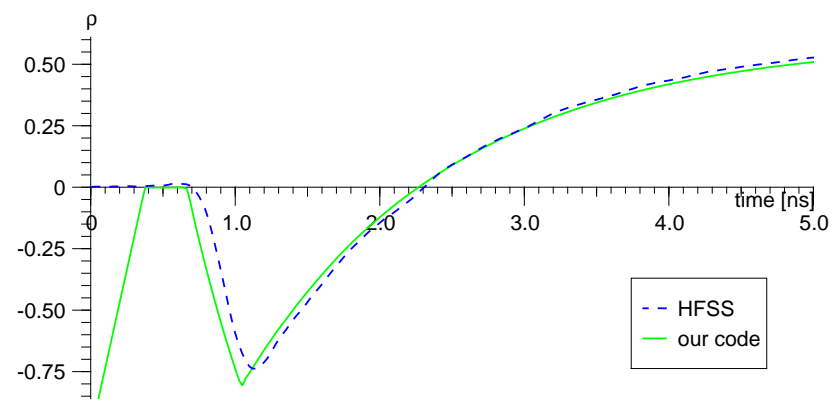

Fig. 7. Calculated TDR trace with HFSS (blue dashed line) and our code (green line) for two-rod probe with length $l=0.1 \mathrm{~m}, D=4 \mathrm{~mm}$, $\kappa=10, \epsilon_{\infty}^{\prime}=1, \sigma=10^{-30} \mathrm{~S} / \mathrm{m}$, not dispersive, parallel resistive capacitive termination, $R_{T}=1436.8 \Omega, C_{T}=5.0 \cdot 10^{-12} \mathrm{~F}$.

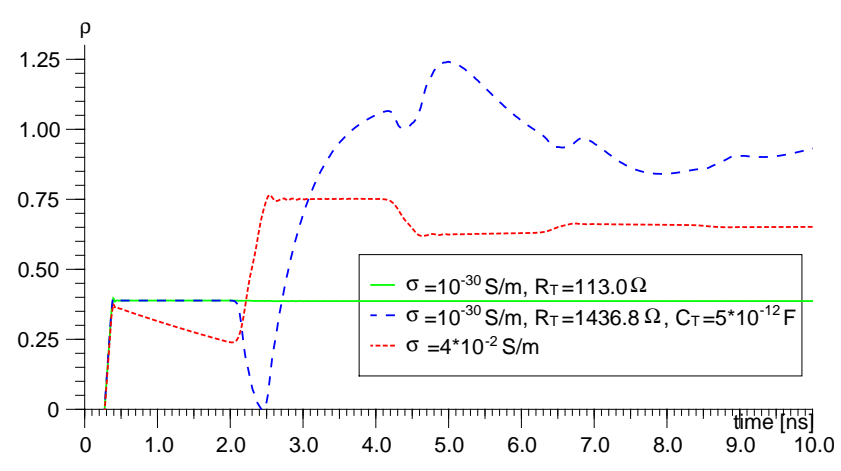

Fig. 8. Calculated TDR trace with our code for two-rod probe with length $l=0.1 \mathrm{~m}, D=4 \mathrm{~mm}, \kappa=10, \epsilon_{\infty}^{\prime}=10$, not dispersive: (i) (green line) $\sigma=10^{-30} \mathrm{~S} / \mathrm{m}$, resistive termination, $R_{T}=113 \Omega$; (ii) (blue dashed line) $\sigma=10^{-30} \mathrm{~S} / \mathrm{m}$, parallel resistive capacitive termination, $R_{T}=1436.8 \Omega, C_{T}=5 \cdot 10^{-12} \mathrm{~F}$; (iii) (red dashed line) $\sigma=4 \cdot 10^{-2} \mathrm{~S} / \mathrm{m}$, infinite termination. The discontinuity of impedance form TDR source to the probe causes the reflection at $0.3 \mathrm{~ns}$.

dotted TDR trace demonstrates the effect of ohmic conductivity between the probe conductors with an open terminated probe.

\subsection{Validation of dispersive dielectric TDR model}

Figures 9 and 10 show the results for TDR traces calculated with dispersive media and open probe termination. At Fig. 9 we compare our code with HFSS ${ }^{\mathrm{TM}}$. There is also a difference between the result of $\mathrm{HFSS}^{\mathrm{TM}}$ and our code. With HFSS $^{\text {TM }}$ we calculate the TDR probe fully 3-dimensional so that the effects of the finite length of the probe are included. Our code, as described above, does not include this effect. Figure 10 shows traces calculated with our code, assuming the TDR source has an impedance of $50 \Omega$. There is an initial relection at $0.3 \mathrm{~ns}$. Effects of different dispersive media can be seen: (i) The amplitude of the reflected signal decreases slowly after the initial reflection. We note that the 
Table 4. Parameters used for hierarchical TDR trace reconstruction of laboratory traces.

\begin{tabular}{lll}
\hline optimization parameter & value to Figs. 12-14 & value to Fig. 15 \\
\hline population size & 50 & 50 \\
crossover probability & 0.6 & 0.05 \\
mutation probability & 0.01 & 0.01 \\
bits for $\epsilon_{r}^{\prime} / \epsilon_{\infty}^{\prime}$ & 20 & 20 \\
bits for $\Delta \epsilon^{\prime}$ & - & 20 \\
bits for $f_{\text {rel }}$ & - & 20 \\
bits for conductivity & 20 & 20 \\
bits for terminal resistor & - & 10 \\
bits for terminal capacitor & - & - \\
transmission line termination & resistive & resistive, optimized \\
termination resistor & $214 \Omega$ & $786.9 \Omega$ \\
TDR rise time $t_{\text {rise }}$ & $28 \mathrm{ps}$ & $30 \mathrm{ps}$ \\
spatial discretization & $0.0005 \mathrm{~m}$ & $0.001 \mathrm{~m}$ \\
time step security & 0.9 & 0.25 \\
TDR probe type & two rod & three rod \\
probe length & $1.0 \mathrm{~m}$ & $0.2 \mathrm{~m}$ \\
conductor diameter $D$ & $10.0 \mathrm{~mm}$ & $4.0 \mathrm{~mm}$ \\
conductor center distance $d$ & $30.8 \mathrm{~mm}$ & $25.0 \mathrm{~mm}$ \\
$\kappa$ & 3.08 & 6.25 \\
\hline
\end{tabular}

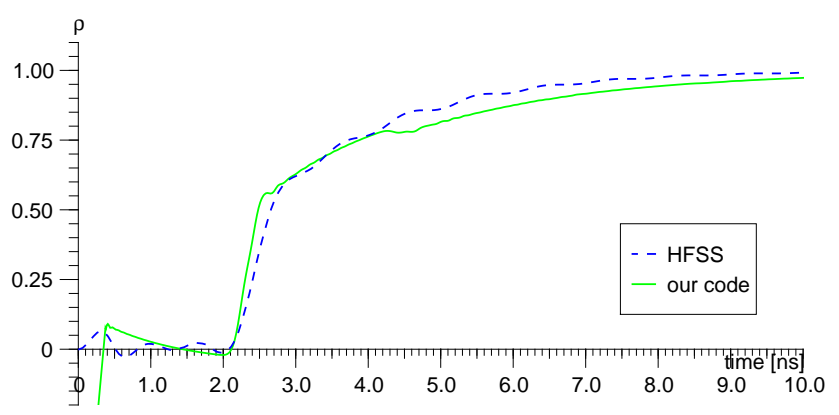

Fig. 9. Calculated TDR trace with HFSS (blue dashed line) and our code (green line) for two-rod probe with length $l=0.1 \mathrm{~m}, D=4 \mathrm{~mm}$, $\kappa=10, \epsilon_{\infty}^{\prime}=10, \sigma=10^{-30} \mathrm{~S} / \mathrm{m}$, infinite termination, dispersive media with $\Delta \epsilon^{\prime}=10, f_{\mathrm{rel}}=100 \mathrm{MHz}$.

ohmic conductivity between the probe rods can be neglected; (ii) the reflections tend to smooth with the increasing effect of dispersion.

\subsection{Hierarchical reconstruction of water content profiles}

\subsubsection{Traces measured in non-dispersive media}

In Figs. 12-14 we show hierarchical reconstructions of the dielectric parameters for the same traces used by Oswald et al. (2003). The probe was in a sand tank with water content $\theta_{1}=\theta_{3}=0$ and $\theta_{2}$ was varied. The experimental setup is sketched in Fig. 11. Relevant optimization parameters are given in Table 4. The vertical dashed lines in fitness and error

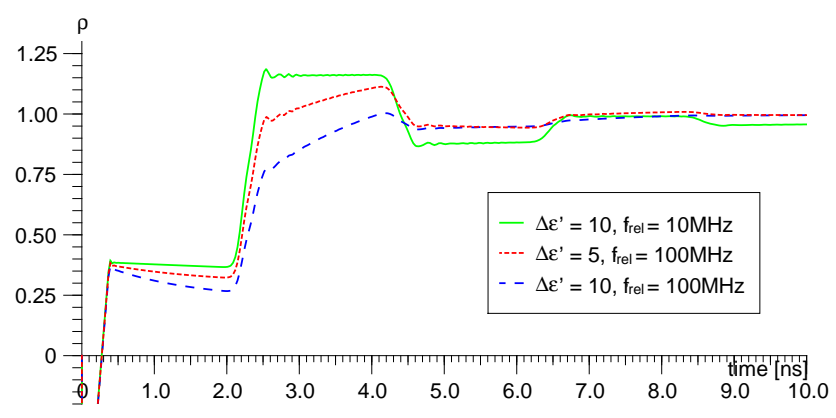

Fig. 10. Calculated TDR trace with our code for two-rod probe with length $l=0.1 \mathrm{~m}, D=4 \mathrm{~mm}, \kappa=10, \epsilon_{\infty}^{\prime}=10, \sigma=10^{-30} \mathrm{~S} / \mathrm{m}$, infinite termination and dispersion: (i) (green line) $\Delta \epsilon^{\prime}=10, f_{\text {rel }}=10 \mathrm{MHz}$; (ii) (red dotted line) $\Delta \epsilon^{\prime}=5, f_{\text {rel }}=100 \mathrm{MHz}$; (iii) (blue dashed line) $\Delta \epsilon^{\prime}=10, f_{\text {rel }}=100 \mathrm{MHz}$. The discontinuity of impedance form TDR source to the probe causes the reflection at $0.3 \mathrm{~ns}$.

history indicate an increase in spatial resolution. The number of spatial intervals are given in red in history and fitness.

For Fig. 12 with water content $\theta_{2}=0$ we see no significant increase in fitness, when increasing the spatial resolution during the optimization. For traces with inhomogeneous water content, Fig. 13: $\theta_{2}=0.05$, Fig. 14: $\theta_{2}=0.10$, we see an increase in fitness, if the spatial resolution is commensurate with the region where the water content varies. The hierarchical reconstruction requires about an order of magnitude less iterations for the same traces as the reconstruction with full spatial resolution right from the optimization's start. Additionally, the hierarchical approach leads to considerably smoother profiles when compared to Oswald et al. (2003). 


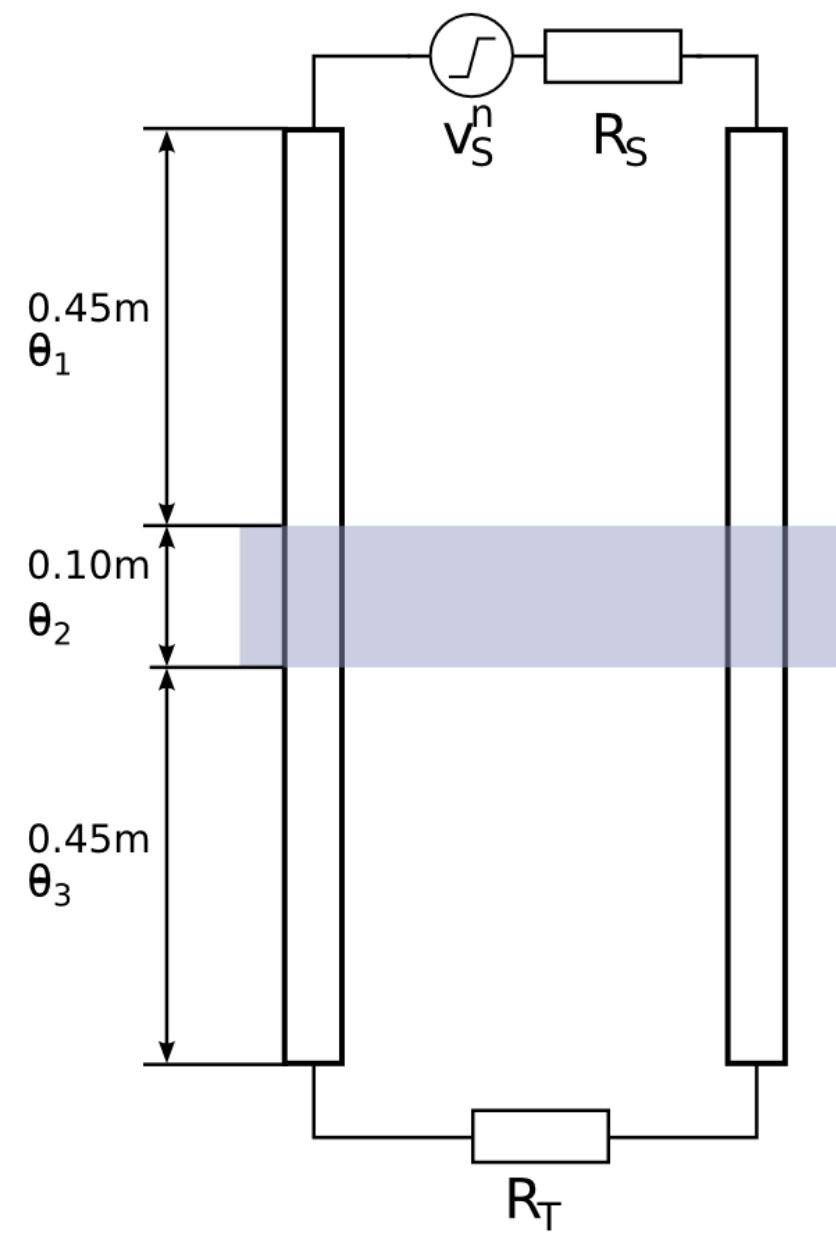

Fig. 11. Experimental setup by Oswald et al. (2003): segmented sand tank with different water contents.

Table 5. Comparison of volumetric measured water content, permittivity of composite with Roth et al. (1990)'s model ( $\alpha=0.5$, $\left.\epsilon_{\text {soil }}=5, \eta=0.322\right)$ and the reconstructed relative dielectric permittivity corresponding to Fig. 15.

\begin{tabular}{ccccc}
\hline $\begin{array}{c}\text { depth }(\mathrm{cm}) \\
\text { volumetric }\end{array}$ & $\theta$ & $\epsilon_{c}$ & $\begin{array}{c}\text { depth }(\mathrm{cm}) \\
\text { reconstructed }\end{array}$ & $\epsilon_{r}+\Delta \epsilon$ \\
\hline \multirow{2}{*}{$0 \ldots 4$} & 0.001 & 3.41 & $0.0 \ldots 2.5$ & 4.32 \\
& & & $2.5 \ldots 5.0$ & 3.99 \\
$5 \ldots 9$ & 0.001 & 3.41 & $\begin{array}{c}7.0 \ldots 7.5 \\
3.5 \ldots 10.0\end{array}$ & 4.33 \\
& & & $10.0 \ldots 12.5$ & 8.72 \\
$10 \ldots 14$ & 0.022 & 4.06 & $12.5 \ldots 15.0$ & 11.31 \\
& & & $15.0 \ldots 17.5$ & 12.44 \\
$15 \ldots 19$ & 0.224 & 13.18 & $17.5 \ldots 20.0$ & 12.16 \\
$20 \ldots 24$ & 0.322 & 19.48 & - & - \\
\hline
\end{tabular}

\subsubsection{Traces measured in dispersive media}

In Fig. 15 we show the hierarchical reconstruction of dispersive dielectric parameters for a TDR trace, measured verti-
Table 6. Parameters used for hierarchical TDR trace reconstruction of field data.

\begin{tabular}{ll}
\hline TDR/optimization parameter & value \\
\hline population size & 50 \\
crossover probability & 0.6 \\
mutation probability & 0.01 \\
bits for $\epsilon_{\infty}^{\prime}$ & 20 \\
bits for $\Delta \epsilon^{\prime}$ & 20 \\
bits for $f_{\text {rel }}$ & 20 \\
bits for conductivity & 20 \\
bits for terminal resistor & 10 \\
bits for terminal capacitor & 10 \\
transmission line termination & parallel resistive \\
& capacitive, optimized \\
TDR rise time $t_{\text {rise }}$ & $460 \mathrm{ps}$ \\
measured samples & 251 \\
time between samples & $107 \mathrm{ps}$ \\
spatial discretization & $0.001 \mathrm{~m}$ \\
time step security & 0.2 \\
TDR probe type & three rod \\
probe length & $0.3 \mathrm{~m}$ \\
conductor diameter $D$ & $4.8 \mathrm{~mm}$ \\
conductor center distance $d$ & $22.5 \mathrm{~mm}$ \\
$\kappa$ & $4.69 \mathrm{~mm}$ \\
\hline
\end{tabular}

cally in a sand tank. The probe ends in fully water saturated sand, while the sand at the probe head is dry. So we expect a strong gradient in the water content along the probe. We measure the water content volumetrically in different depths (probe head corresponds to $z=0.0 \mathrm{~m}$ ). If we compare the reconstructed dielectric parameters with volumetrically measured parameters at Table 5, we see that they are in the same order; resistivity and permittivity increase in the longitudinal direction (for this comparison we use the relative dielectric permittivity $\epsilon_{s}^{\prime}=\epsilon_{\infty}^{\prime}+\Delta \epsilon^{\prime}$ ). Only the volumetric measurement at depth $10 \ldots 14 \mathrm{~cm}$ and the reconstructed value at $10.0 \ldots 12.5 \mathrm{~cm}$ do not correspond. We denote that the different depths are not quite compatible: there is an uncertainty in the depth of the volumetric measurement and the TDR probe, is not calibrated.

In the discretized voltage update procedure, Eq. (22) the parameters describing the dispersion appear only as products. If one of the reconstructed dispersion parameters $f_{\text {rel }}$ or $\Delta \epsilon^{\prime}$ approaches zero, the other dispersion parameter has no effect and the result for it is not relevant. For example in Fig. 15 we get for $x=0 \ldots .2 .5 \mathrm{~cm}$ a relaxation frequency of round about $200 \mathrm{MHz}$. That does not mean, that the sand there has this relaxation frequency, because the $\Delta \epsilon^{\prime}$ is nearly zero. In addition, the relaxation frequency $f_{\text {rel }}$ of water saturated sand is in the range of some GHz (Robinson et al., 2003). So the Debye model for dispersion has no strong gradient in dielectric permittivity in the most relevant frequency 

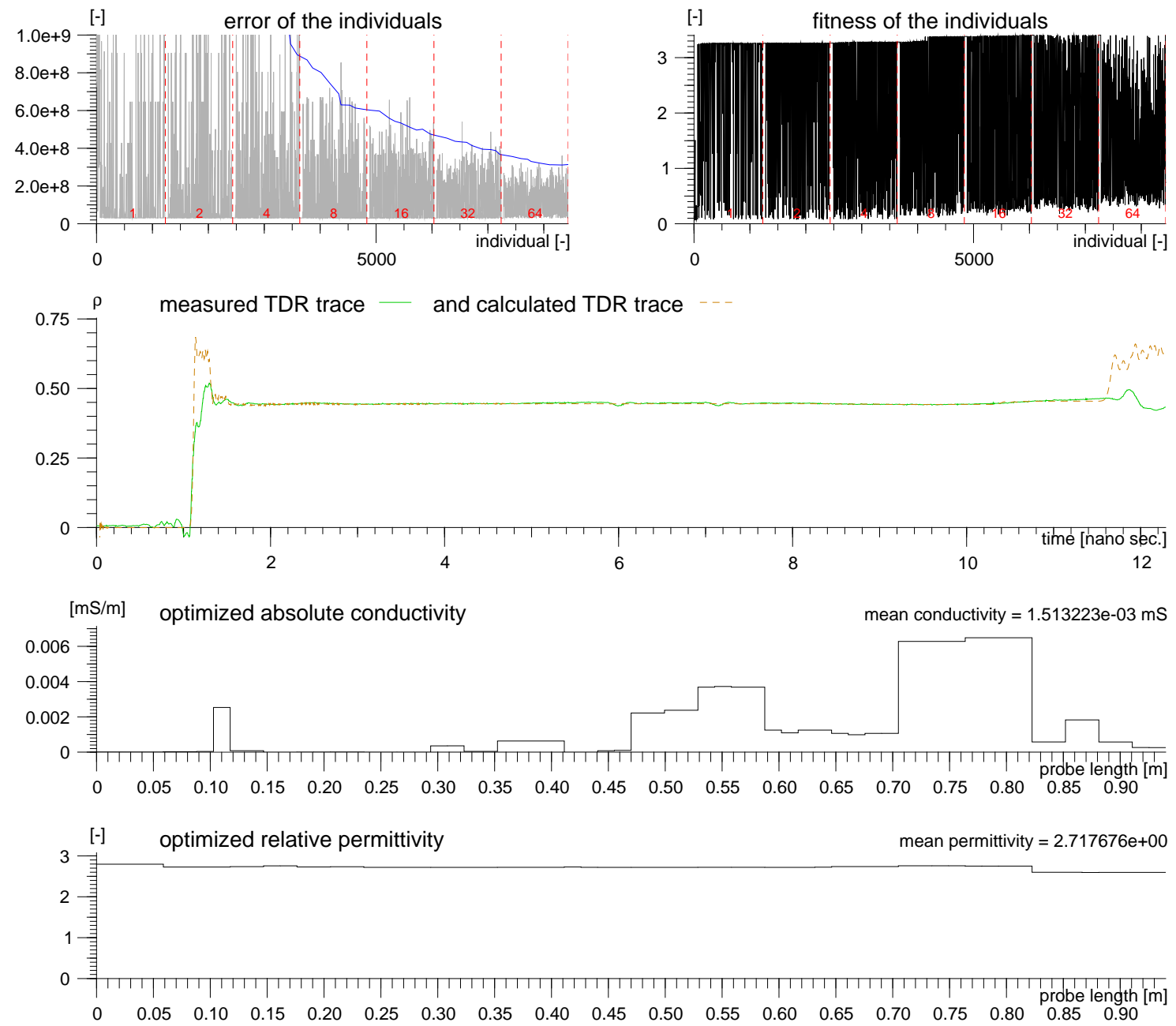

Fig. 12. Reconstruction of synthetic profile, $\theta_{2}=0$. Individual 7547 with error: $2.9 \cdot 10^{7}$ and fitness: 3.4 . Effective relative permittivity $\epsilon_{c}$ corresponding to $\theta_{2}$ : (i) travel time evaluation $\epsilon_{c}=2.78$; (ii) Roth et al. (1990)'s model $\epsilon_{c}=2.49$.

range of our TDR. It is not easy to extrapolate the relaxation frequency very well and we get large errors for it with the frequency range of our TDR. Nevertheless the dispersion can not be neglected. Inclusion of dielectric dispersion is required to invert this TDR trace; reconstruction without dispersion will inevitably fail.

\subsubsection{Traces measured under field conditions}

In Figs. 16-19 we show hierarchical reconstructions of TDR traces measured under field condition at the Grenzhof (Heidelberg, Germany) test site (Wollschläger and Roth, 2005). The traces were recorded with a "Campbell TDR 100" using a Campbell probe "CS610". Essential TDR properties and the parameters used in the optimization to produce Figs. 1619 are shown in Table 6. The steps in all these measured traces result from finite time resolution in recording. The first reflection in all traces is a result of the TDR probe head. The head is simulated with a transmission line section. Gen- erally, we can fit the transmission line parameters for this part with our simulation. Because the parameters are constant for every single probe, we fit them manually and fix the respective parameters in the job file, because it would unnecessarily slow down the optimization if it was fitted for every trace from scratch once again. We particularly note Fig. 16. More conventional techniques (e.g. Roth et al., 1990) experience severe problems, may even fail, to evaluate this trace, because there is no sharp reflection from the end of probe.

\section{Discussion}

We have derived analytical expressions for the transmission line parameters of a three rod probe, based on an approximate model and validated it with numerical simulations; such expressions, to our knowledge, have not been presented in the area of the TDR literature yet. The analytical model has been benchmarked against results obtained from an established 

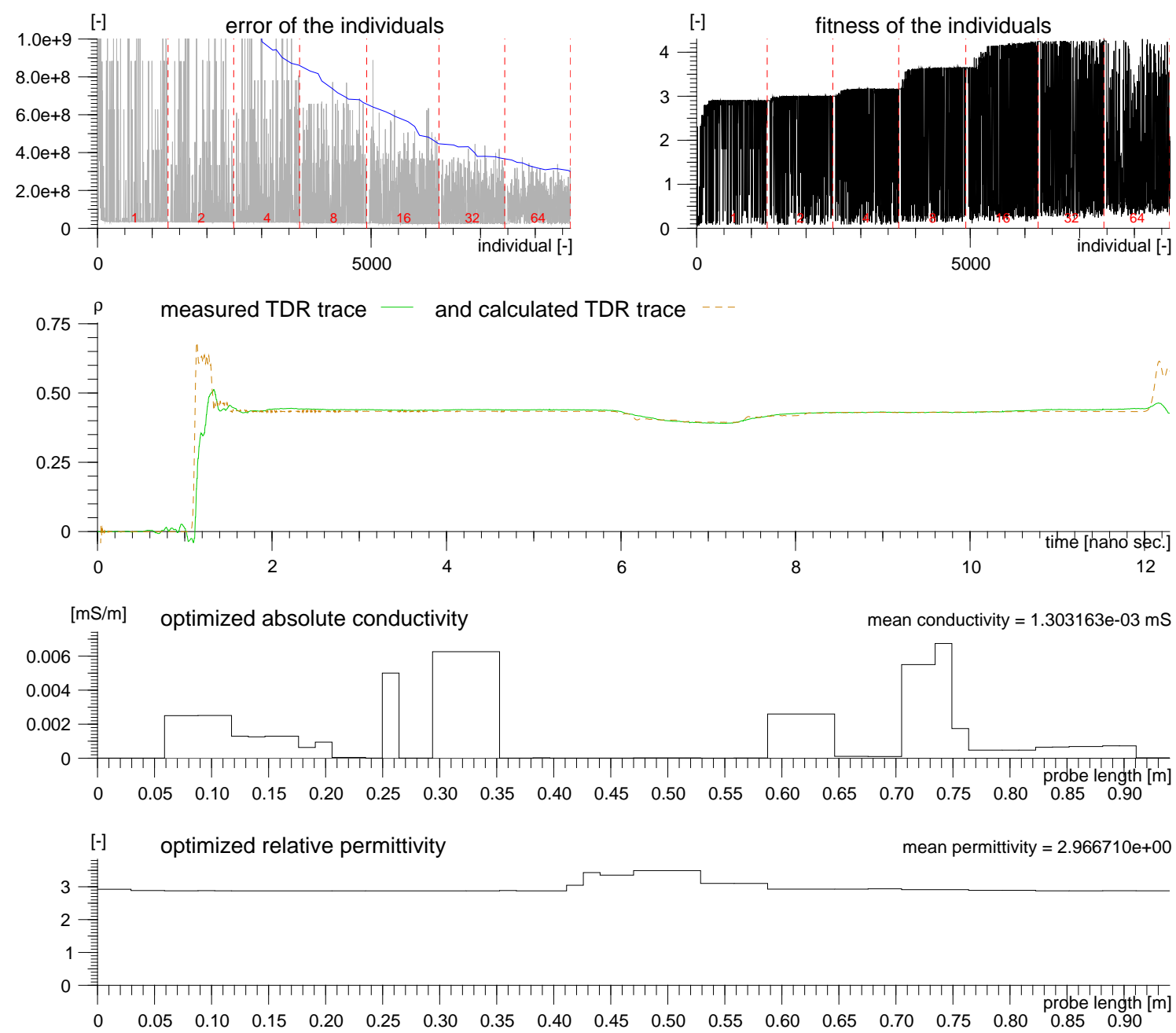

Fig. 13. Reconstruction of synthetic profile, $\theta_{2}=0.05$. Individual 8146 with error: $2.3 \cdot 10^{7}$ and fitness: 4.3 . Effective relative permittivity $\epsilon_{c}$ corresponding to $\theta_{2}$ : (i) travel time evaluation $\epsilon_{c}=3.57$; (ii) Roth et al. (1990)'s model $\epsilon_{c}=3.90$.

commercial solver. We mention that the analytical model is most accurate for larger $\kappa$ and becomes less accurate at very low $\kappa$. This is caused by the fact that in the situation of large, closely spaced probe rods the electric and magnetic fields, obtained from the assumption of infinitely thin and infinitely extended line charges and current filaments, respectively, increasingly differ from the true fields. Nevertheless, it is remarkable how accurate its predictions are at larger $\kappa$.

We have validated our dispersive TDR code by comparing synthetically calculated traces from our code and HFSS ${ }^{\mathrm{TM}}$, for both dispersive and non-dispersive dielectric properties. We mention that dispersive dielectrics impose more stringent restrictions onto the time-step of the explicit integration scheme to keep it stable. This is related to the relative position of the dielectric relaxation frequency and the dominant frequency content of the TDR signal source.

We have used a hierarchical approach to reconstruct electric parameter profiles from TDR traces measured in the lab- oratory with minimal electrical losses. The hierarchical approach reduced the number of forward solutions required and leads to considerably smoother profiles. We consider hierarchical optimization to be a definite advance and speculate that this will hopefully support the deployment of TDR profile reconstruction to field applications. The reconstructed profiles shown in the paper are computed on a personal computer ( $2 \mathrm{GHz}$ clock) within a few hours. The actual computation time depends strongly on the measurement configuration, e.g. probe length, TDR rise time, dispersive character, upper and lower limits for the reconstructed parameters. The rather massive computational effort results from the requirement to employ a globally optimizing algorithm since this type of problem is often vulnerable to local minima. We chose a genetic algorithm which is known to be very robust. Algorithmic improvements are easily accomplished, for instance by using hybrid approaches, but they were not deemed essential for this prove-of-concept study. 

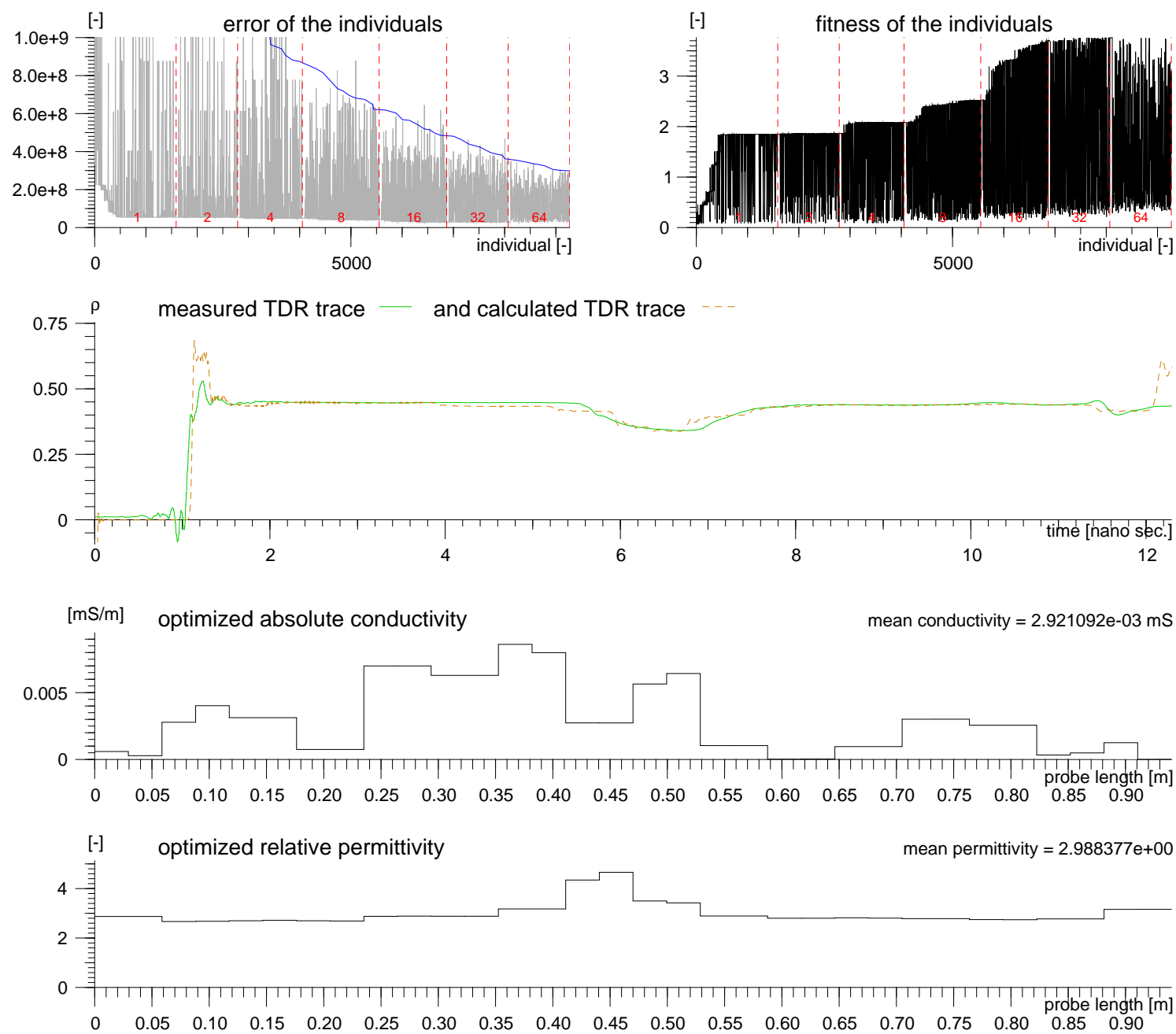

Fig. 14. Reconstruction of synthetic profile, $\theta_{2}=0.10$. Individual 7977 with error: $2.7 \cdot 10^{7}$ and fitness: 3.8 . Effective relative permittivity $\epsilon_{c}$ corresponding to $\theta_{2}$ : (i) travel time evaluation $\epsilon_{c}=5.09$; (ii) Roth et al. (1990)'s model $\epsilon_{c}=5.64$.

Numerical experimentation for reconstructing TDR traces measured in the field has definitely shown that dispersive dielectric properties must be included in the numerical model. Only when using dispersive dielectrics can such TDR traces be recovered numerically; using frequency-independent permittivity alone can not account for the the shape of the traces.

If the frequency range of the TDR instrument is well below the relaxation frequency, dispersion becomes less important. On the other hand, if the TDR's frequency content and the relaxation frequency have a significant overlap, then dispersion will be quite pronounced. The "Campbell TDR 100" has $f_{3 \mathrm{~dB}} \approx 740 \mathrm{MHz}$. The relaxation frequencies extracted by the optimization are within this range and therefore dispersion is relevant (Robinson et al., 2003, 2005).

We note that in all cases we used a relatively small mutation probability, 0.01 , and a significantly higher cross-over probability, 0.6. Increasing the mutation probability results in a more diverse population but does not seem to accelerate the convergence behavior. On the other hand, using a relatively high cross-over probability ensures efficient reconstruction. The error and fitness histories represent the search in a wide parameter range. For some individuals we obtain a high error and a low fitness, respectively. The low fitness of some individuals give the black filled area in fitness history. Note that the error and fitness history are line plots. The high errors are cut off in the plots so that the relevant sector is visible. Additionally, the error's running average is plotted in the diagrams with a blue line.

Furthermore, a more realistic numerical boundary condition using a parallel resistive-capacitive impedance is essential. Using all these model components we succeed in reconstructing field measured TDR traces over a wide spectrum of dielectric permittivity and conductivity. We note that dielectric loss caused by the dispersive Debye model is fundamentally different from ohmic loss. We finally mention that our profile reconstruction does not require any a priori information whatsoever in order to succeed. 

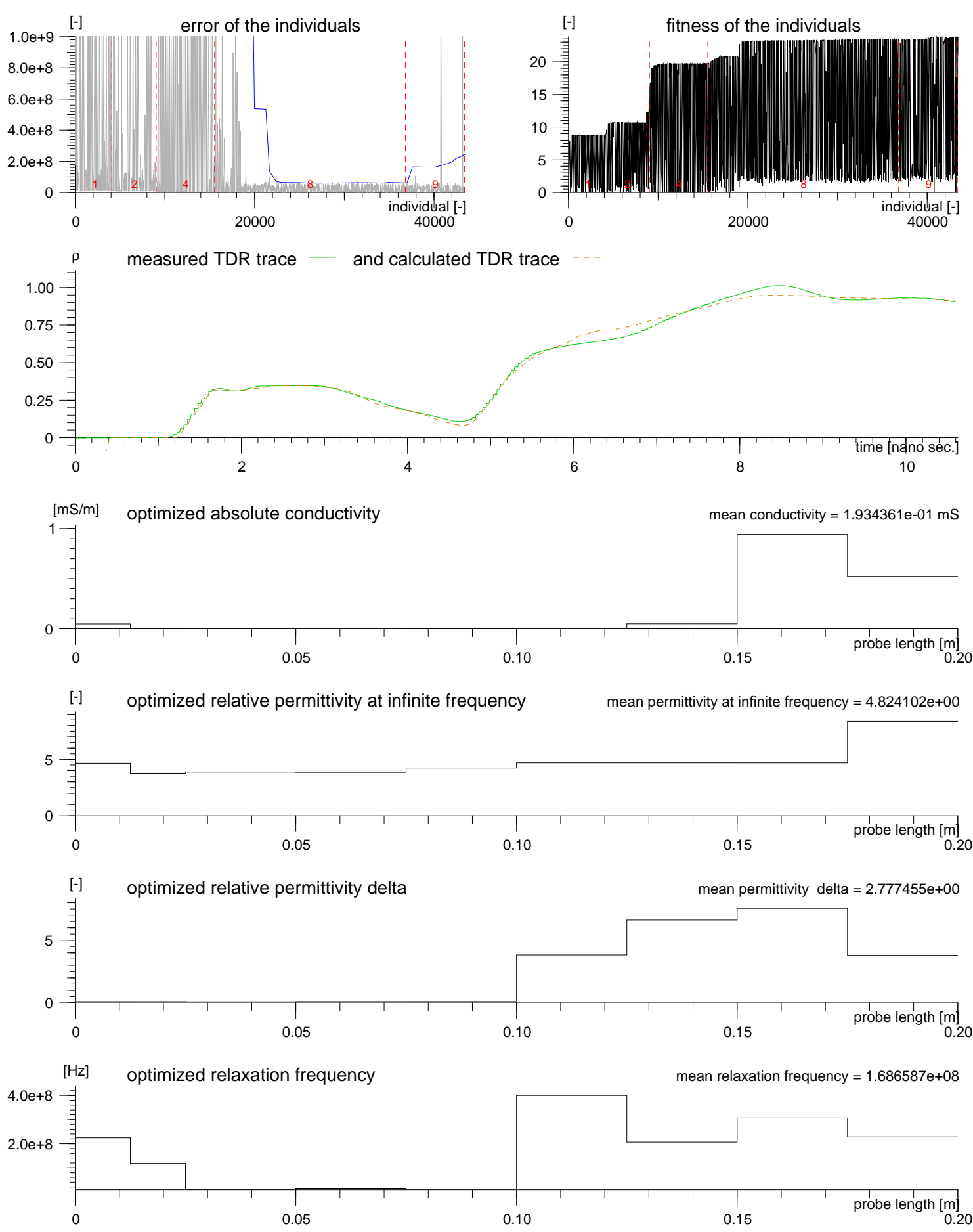

Fig. 15. Reconstruction of profile with strong gradient. TDR probe vertical in sand tank. Sand at probe's head dry, at probe's end fully water saturated. Individual 43402 with error: $4.2 \cdot 10^{6}$, fitness: 28 , and terminal impedance: $650 \Omega$. For the discussion of the dispersive dielectric parameters see Sect. 3.3.2. 

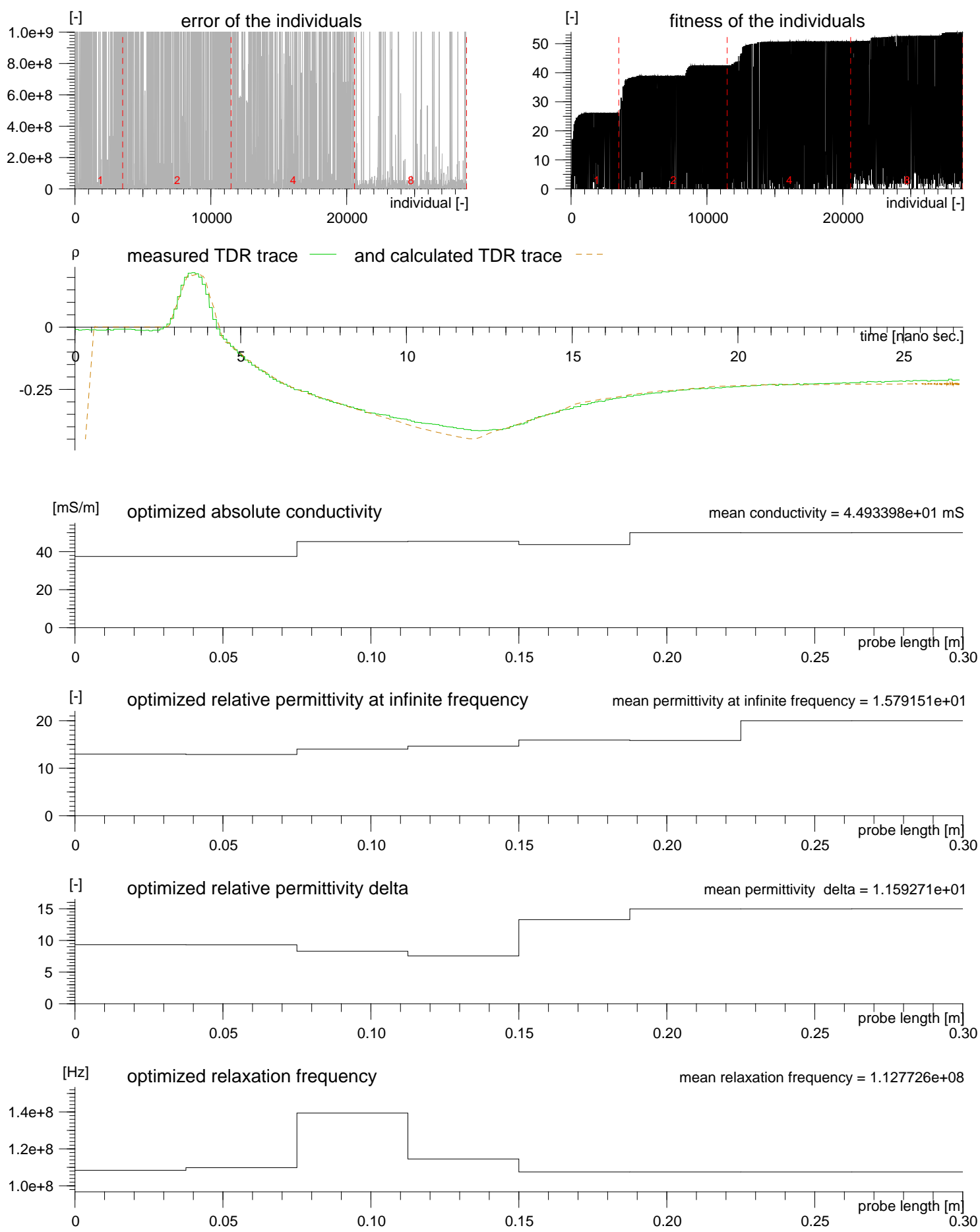

Fig. 16. Reconstructed TDR trace, measured at Grenzhof, Heidelberg, Germany in $1.41 \mathrm{~m}$ depth. Individual 28795 with error: $1.9 \cdot 10^{6}$, fitness: 54 , terminal impedance: $305 \Omega$, and terminal capacitance $3 \cdot 9 \cdot 10^{-17}$ F. For the discussion of the dispersive dielectric parameters see Sect. 3.3.2. 

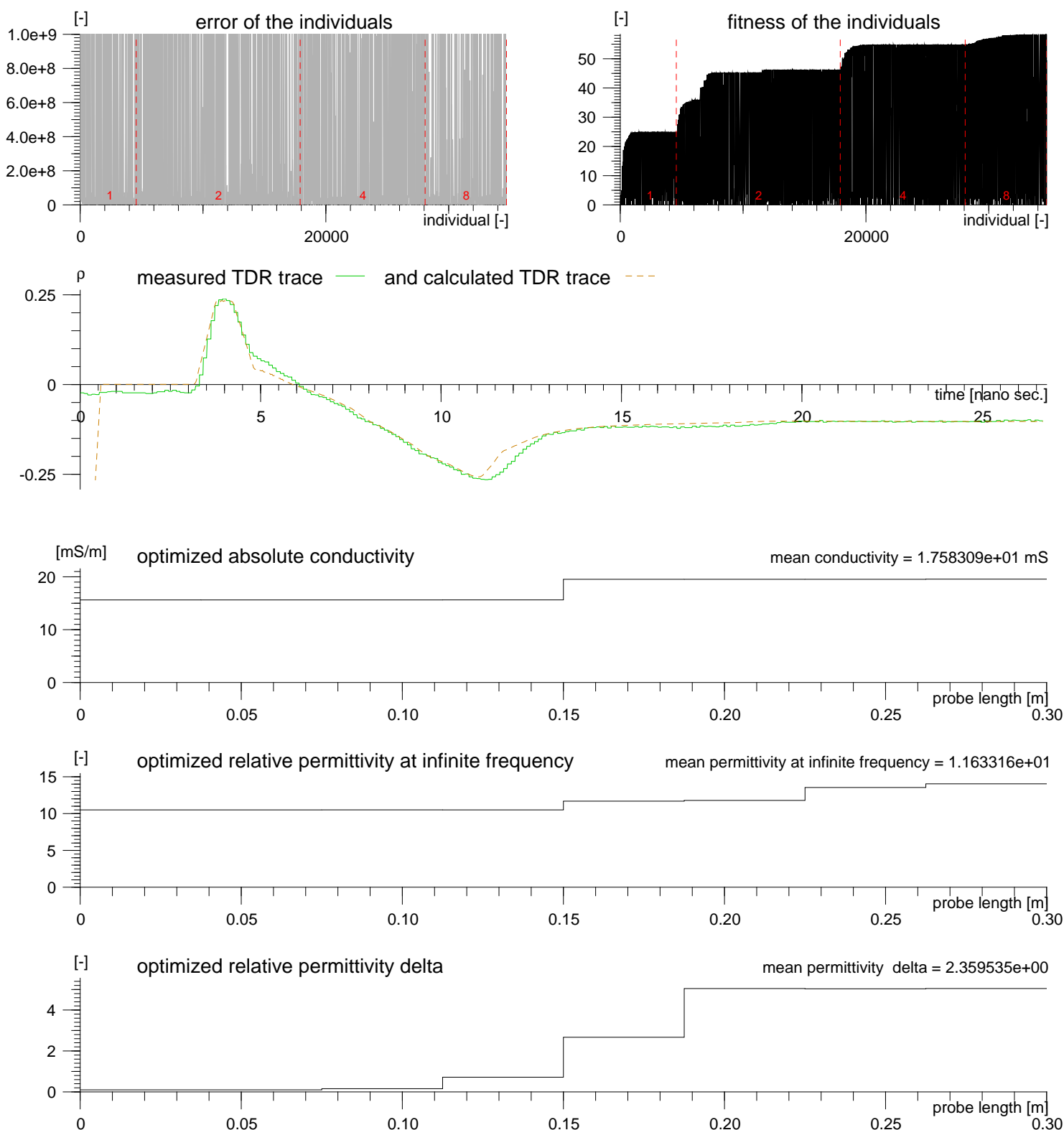

$[\mathrm{Hz}] \quad$ optimized relaxation frequency $\quad$ mean relaxation frequency $=1.832296 \mathrm{e}+08$

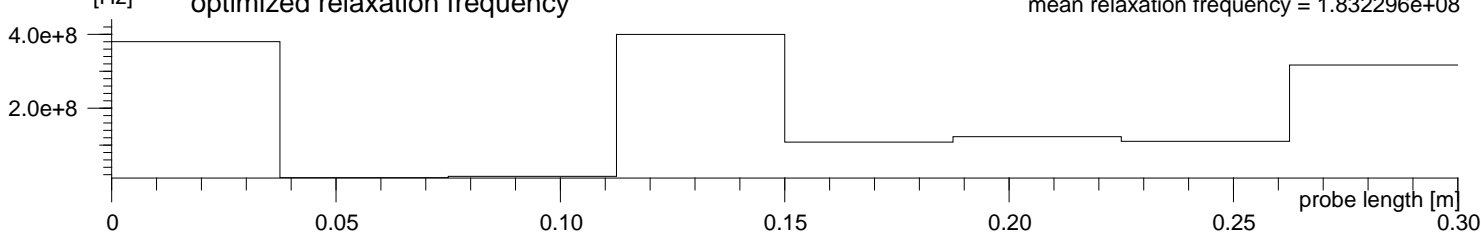

Fig. 17. Reconstructed TDR trace, measured at Grenzhof, Heidelberg, Germany in $0.72 \mathrm{~m}$ depth. Individual 34702 with error: $1.7 \cdot 10^{6}$, fitness: 58, terminal impedance: $740 \Omega$, and terminal capacitance $2 \cdot 0 \cdot 10^{-17} \mathrm{~F}$. For the discussion of the dispersive dielectric parameters see Sect. 3.3.2. 

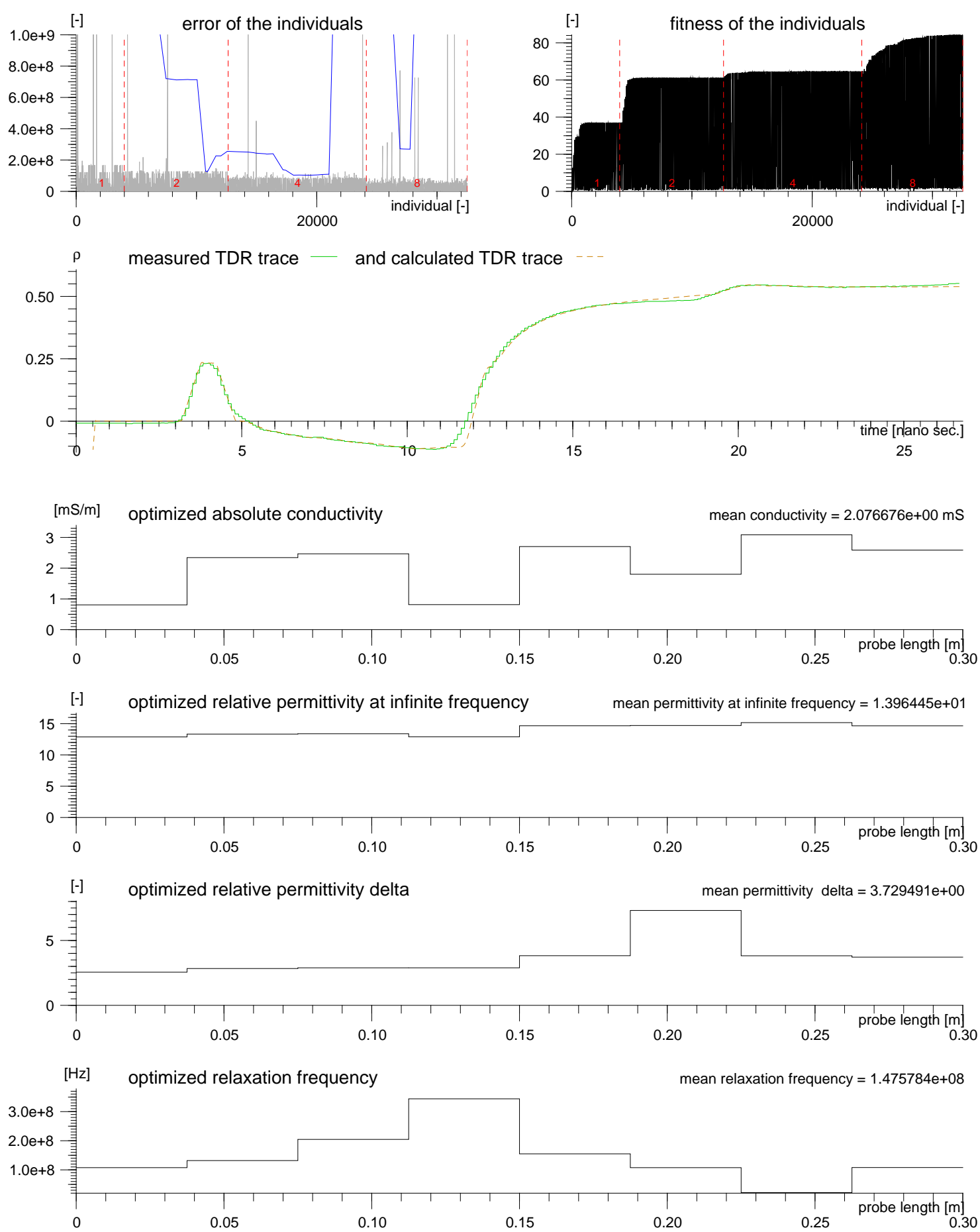

Fig. 18. Reconstructed TDR trace, measured at Grenzhof, Heidelberg, Germany in $0.13 \mathrm{~m}$ depth. Individual 32504 with error: $1.2 \cdot 10^{6}$, fitness: 84 , terminal impedance: $216 \Omega$, and terminal capacitance $1.8 \cdot 10^{-18} \mathrm{~F}$. For the discussion of the dispersive dielectric parameters see Sect. 3.3.2. 

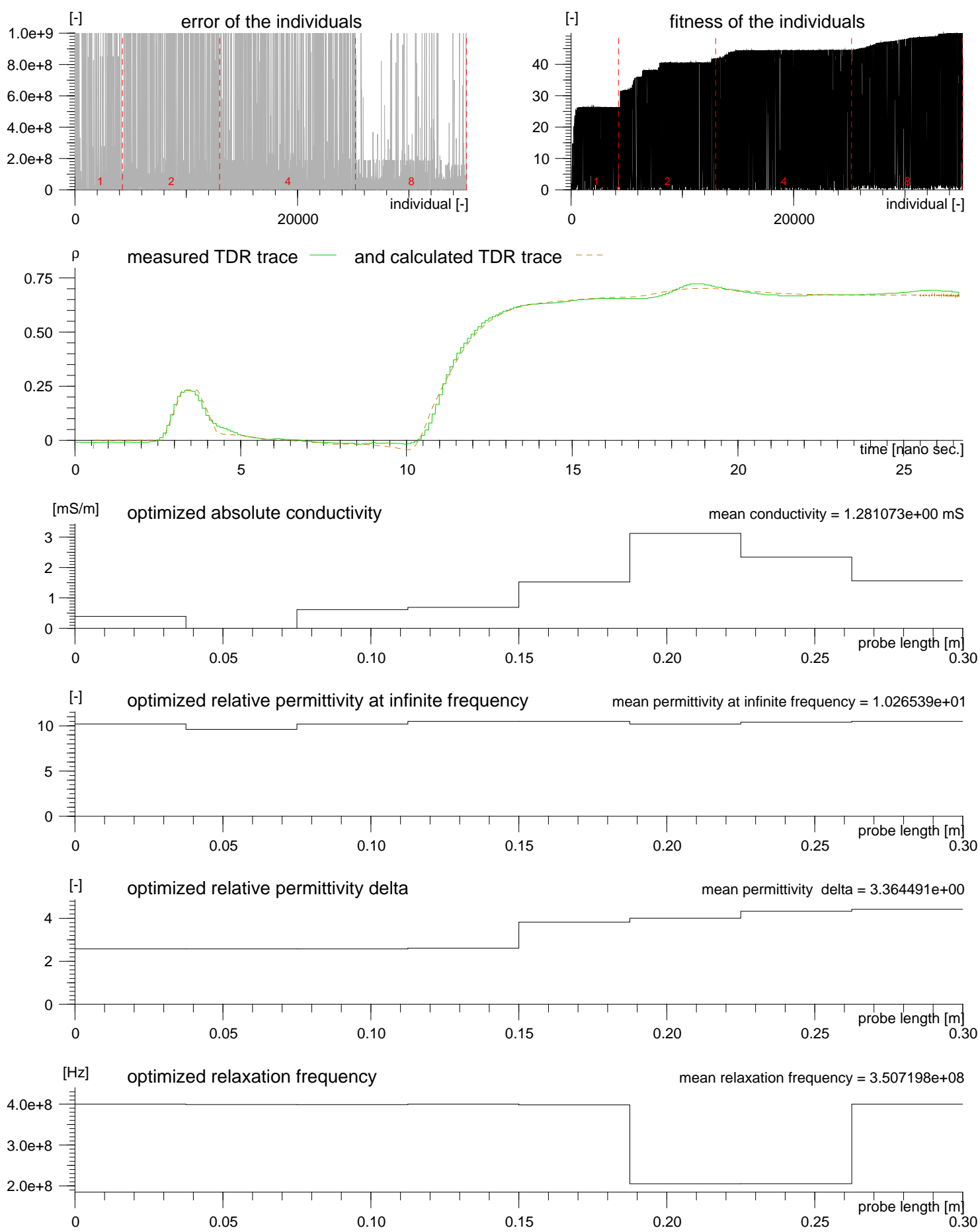

Fig. 19. Reconstructed TDR trace, measured at Grenzhof, Heidelberg, Germany in $0.30 \mathrm{~m}$ depth. Individual 35116 with error: $2.0 \cdot 10^{6}$, fitness: 50 , terminal impedance: $315 \Omega$, and terminal capacitance $5 \cdot 6 \cdot 10^{-19}$ F. For the discussion of the dispersive dielectric parameters see Sect. 3.3.2. 


\section{Conclusions}

A robust, accurate and efficient method has been presented for reconstructing dielectric and ohmic conductivity profiles along TDR traces, for both laboratory and field traces. Different boundary conditions have been implemented for modeling a wide variety of probe terminations encountered in experimental setups. Dispersive dielectric properties are reconstructed and may be of interest for extracting even more information from TDR traces, such as a distinction between bound and free water, so characteristical for clay and loam soils (Ishida et al., 2000).

Now, that TDR technology using conventional, transverseelectric-magnetic (TEM) probes has reached considerable maturity we speculate that it could be worthwhile to address more advanced concepts, such as the single-rod probe using a transverse-magnetic mode of propagation, (Oswald et al., 2004; Nussberger et al., 2005). Such probe types may pose modeling challenges but they also hold the promise of avoiding problems of probes with multiple conducting rods.

The code developed in this work will be publicly available at http://www.iup.uni-heidelberg.de/institut/forschung/ groups/ts/tools in due course.

\section{Appendix A}

\section{Three-rod probe transmission line parameters}

The electric potential of a line charge, with diameter $D$, in $x$-direction, cf. Fig. 1, outside the conductor is given by

$\Phi_{e l}(y, z)=\Phi_{0}-\frac{Q}{\ell} \frac{1}{2 \pi \epsilon} \ln \left(\sqrt{y^{2}+z^{2}}\right)$

with potential $\Phi_{0}$ at infinity and line charge density $\frac{Q}{\ell}$. By convention, the potential at infinity is set to zero. We consider three parallel, infinitely long line charges, Fig. 1. The total potential, outside the conductors, is the superposition of of the single rod potential, Eq. (A1):

$$
\begin{aligned}
\Phi_{e l}= & \frac{Q}{\ell} \frac{1}{2 \pi \epsilon}\left\{\frac{1}{2} \ln \left[\left(y^{2}+(z-d)^{2}\right)\left(y^{2}+(z+d)^{2}\right)\right]\right. \\
& \left.-\ln \left[y^{2}+z^{2}\right]\right\} .
\end{aligned}
$$

The capacitance per unit length between conductor 0 and 1 is

$C_{01}^{\prime}=\frac{\frac{Q}{\ell}}{V}$

with the potential difference $V$ between the two nearest points of conductor 0 and $1:\left(y=0, z=\frac{D}{2}\right)$ and $\left(y=0, z=d-\frac{D}{2}\right)$.

$V=\Phi_{\mathrm{el}}\left(y=0, z=\frac{D}{2}\right)-\Phi_{e l}\left(y=0, z=d-\frac{D}{2}\right)$

www.hydrol-earth-syst-sci.net/10/209/2006/

$$
=\frac{Q}{\ell} \frac{1}{2 \pi \epsilon}\left[\ln \left(\frac{4 d^{2}-D^{2}}{4 d D-D^{2}}\right)+\ln \left(\frac{2 d-D}{D}\right)\right] .
$$

Due to the symmetry of the conductor arrangement the capacitance of a three-rod probe is twice the capacitance, resulting from Eq. (A4). Therefore, the capacitance per unit length is

$$
C^{\prime}=\frac{4 \pi \epsilon}{\ln \left(\frac{4 d^{2}-D^{2}}{4 d D-D^{2}}\right)+2 \ln \left(\frac{2 d-D}{D}\right)} .
$$

The conductance per unit length $G^{\prime}$ of the medium between the rods is calculated from the electric potential. We use Ohm's law

$\boldsymbol{j}=\sigma \boldsymbol{E}$

with current density $\boldsymbol{j}$, ohmic conductivity $\sigma$ and the electric field $\boldsymbol{E}=-\nabla \Phi_{e l}$. The current between conductor 0 and 2 per length $\ell$ is the integral of $\boldsymbol{j} \cdot F_{1}$ with $F_{1} \perp z$-axis:

$$
\begin{aligned}
I & =\int_{0}^{\ell} \int_{-\infty}^{+\infty} j_{z} d y d x \\
& =\sigma \ell \int_{-\infty}^{+\infty} E_{z} d y .
\end{aligned}
$$

Using the electric potential, Eq. (A2), and evaluating the integral at $z=-\frac{d}{2}$ we obtain

$I=\frac{\sigma}{\epsilon} Q$.

With the potential difference, Eq. (A4), we compute the conductivity per unit length between conductor 0 and 2 :

$G_{02}^{\prime}=\frac{I^{\prime}}{V}$.

Again, due to the symmetry of the conductor arrangement, Fig. 1, the conductivity per unit length of the three-rod TDR probe is twice $G_{02}^{\prime}$ :

$G^{\prime}=\frac{4 \pi \sigma}{\ln \left(\frac{4 d^{2}-D^{2}}{4 d D-D^{2}}\right)+2 \ln \left(\frac{2 d-D}{D}\right)}$.

The absolute value of the magnetic field outside a wire infinitely, extended in $x$-direction with radius $\frac{D}{2}$, conducting current I, using the definition $r=\sqrt{y^{2}+z^{2}}$ is

$r \geq \frac{D}{2}: \quad|\boldsymbol{B}(r)|=\frac{\mu_{0} I}{2 \pi r}$.

The magnetic induction outside a wire for the three-rod probe is given as a superposition of Eq. (A11)

$$
\begin{aligned}
|\boldsymbol{B}(y, z)|= & \frac{\mu_{0} I}{2 \pi}\left(\frac{2}{\sqrt{y^{2}+z^{2}}}+\frac{1}{\sqrt{y^{2}+(d-z)^{2}}}\right. \\
& \left.-\frac{1}{\sqrt{y^{2}+(d+z)^{2}}}\right)
\end{aligned}
$$


where we have implicitly assumed that we only need the field in a plane parallel to the line connecting the centers of the three conductors, hereby ensuring that the directions of the three magnetic induction components are all parallel. For the inductance only the magnetic flux $\Phi_{m}$ outside the wires is relevant. With Eq. (A12) the magnetic flux through the area $F_{2} \perp y$-axes with $F_{2}=(d-D) \cdot \ell$ at $y=0$ is

$$
\begin{aligned}
\Phi_{m} & =\ell \int_{\frac{D}{2}}^{d-\frac{D}{2}} B_{y}(z) d z \\
& =\frac{\mu_{0} I \ell}{2 \pi}\left[3 \ln \left(\frac{2 d-D}{D}\right)+\ln \left(\frac{2 d+D}{4 d-D}\right)\right] .
\end{aligned}
$$

The self inductance per unit length between conductor 0 and 1 is

$L^{\prime}=\frac{\frac{\Phi_{m}}{\ell}}{I}$.

Due to the symmetry of the arrangement the inductance of the three rod probe is one half the inductance that follows from the magnetic flux Eq. (A14). So the inductance per unit length is

$L^{\prime}=\frac{3 \mu_{0}}{4 \pi}\left[\ln \left(\frac{2 d-D}{D}\right)+\frac{1}{3} \ln \left(\frac{2 d+D}{4 d-D}\right)\right]$.

\section{Appendix B}

\section{Discretization of dispersive dielectric medium}

To obtain the update procedure for the voltage we insert Eq. (21) into Eq. (2):

$$
\begin{aligned}
\frac{\partial i}{\partial x}= & -\left(G^{\prime}+C^{\prime}(t) \otimes \frac{\partial}{\partial t}\right) v \\
= & -G^{\prime} v-C_{0}^{\prime}\left[\left(\epsilon_{\infty}^{\prime} \delta(t)+\frac{\Delta \epsilon^{\prime}}{\tau} e^{-\frac{t}{\tau}} U(t)\right) \otimes \frac{\partial v}{\partial t}\right] \\
= & -G^{\prime} v-C_{0}^{\prime} \epsilon_{\infty}^{\prime} \int_{-\infty}^{+\infty} \frac{\partial v\left(t^{\prime}\right)}{\partial t^{\prime}} \delta\left(t-t^{\prime}\right) d t^{\prime} \\
& -C_{0}^{\prime} \frac{\Delta \epsilon^{\prime}}{\tau} \int_{-\infty}^{+\infty} e^{-\frac{t-t^{\prime}}{\tau}} U\left(t-t^{\prime}\right) \frac{\partial v\left(t^{\prime}\right)}{\partial t^{\prime}} d t^{\prime} .
\end{aligned}
$$

The second term of Eq. (B1) is

$$
C_{0}^{\prime} \epsilon_{\infty}^{\prime} \int_{-\infty}^{+\infty} \frac{\partial v\left(t^{\prime}\right)}{\partial t^{\prime}} \delta\left(t-t^{\prime}\right) d t^{\prime}=C_{0}^{\prime} \epsilon_{\infty}^{\prime} \frac{\partial v(t)}{\partial t} .
$$

The integral of the third term leads, using partial integration, to

$\int_{-\infty}^{+\infty} e^{-\frac{t-t^{\prime}}{\tau}} U\left(t-t^{\prime}\right) \frac{\partial v\left(t^{\prime}\right)}{\partial t^{\prime}} d t^{\prime}=\int_{-\infty}^{t} e^{-\frac{t-t^{\prime}}{\tau}} \frac{\partial v\left(t^{\prime}\right)}{\partial t^{\prime}} d t^{\prime}$

$$
\begin{aligned}
& =\left[e^{-\frac{t-t^{\prime}}{\tau}} v\left(t^{\prime}\right)\right]_{t^{\prime}=-\infty}^{t^{\prime}=t}-\int_{-\infty}^{t} \frac{1}{\tau} e^{-\frac{t-t^{\prime}}{\tau}} v\left(t^{\prime}\right) d t^{\prime} \\
& =v(t)-\frac{1}{\tau} \int_{-\infty}^{t} e^{-\frac{t-t^{\prime}}{\tau}} v\left(t^{\prime}\right) d t^{\prime} .
\end{aligned}
$$

We agree on the following abbreviation:

$\psi(t):=\int_{-\infty}^{t} e^{-\frac{t-t^{\prime}}{\tau}} v\left(t^{\prime}\right) d t^{\prime}$.

We finally obtain the transmission line Eq. (2) for a Debye medium

$$
\begin{aligned}
\frac{\partial i(t)}{\partial x}= & -G^{\prime} v(t)-C_{0}^{\prime} \epsilon_{\infty}^{\prime} \frac{\partial v(t)}{\partial t}-C_{0}^{\prime} \frac{\Delta \epsilon^{\prime}}{\tau} v(t) \\
& +C_{0}^{\prime} \frac{\Delta \epsilon^{\prime}}{\tau^{2}} \psi(t) .
\end{aligned}
$$

The discretized version of $\psi$ is

$$
\begin{aligned}
& \psi_{k}^{n}=\left.\psi(t)\right|_{x_{k}, t_{n}} \\
& =\int_{-\infty}^{n \Delta t} e^{-\frac{n \Delta t-t^{\prime}}{\tau_{k}}} v_{k}\left(t^{\prime}\right) d t^{\prime} \\
& =\int_{-\infty}^{n \Delta t} e^{-\frac{n \Delta t}{\tau_{k}}} e^{\frac{t^{\prime}}{\tau_{k}}} v_{k}\left(t^{\prime}\right) d t^{\prime} \\
& =e^{-\frac{n \Delta t}{\tau_{k}}}\left(\int_{-\infty}^{(n-1) \Delta t} e^{\frac{t^{\prime}}{\tau_{k}}} v_{k}\left(t^{\prime}\right) d t^{\prime}\right. \\
& \left.+\int_{(n-1) \Delta t}^{n \Delta t} e^{\frac{t^{\prime}}{\tau_{k}}} v_{k}\left(t^{\prime}\right) d t^{\prime}\right) \\
& =e^{-\frac{\Delta t}{\tau_{k}}} e^{-\frac{(n-1) \Delta t}{\tau_{k}}}\left(\int_{-\infty}^{(n-1) \Delta t} e^{\frac{t^{\prime}}{\tau_{k}}} v_{k}\left(t^{\prime}\right) d t^{\prime}\right. \\
& \left.+\int_{(n-1) \Delta t}^{n \Delta t} e^{\frac{t^{\prime}}{\tau_{k}}} v_{k}\left(t^{\prime}\right) d t^{\prime}\right)
\end{aligned}
$$

With these expansions we write the first integral as a function of $\psi_{k}^{n-1}$ and the second integral is evaluated using the trapezoidal rule.

$$
\begin{aligned}
\psi_{k}^{n}= & e^{-\frac{\Delta t}{\tau_{k}}} \psi_{k}^{n-1}+\frac{1}{2} e^{-\frac{\Delta t}{\tau_{k}}} e^{-\frac{(n-1) \Delta t}{\tau_{k}}} \Delta t \\
& \cdot\left(e^{\frac{n \Delta t}{\tau_{k}}} v_{k}^{n}+e^{\frac{(n-1) \Delta t}{\tau_{k}}} v_{k}^{n-1}\right) \\
= & e^{-\frac{\Delta t}{\tau_{k}}} \psi_{k}^{n-1}+\frac{\Delta t}{2}\left(v_{k}^{n}+e^{-\frac{\Delta t}{\tau_{k}}} v_{k}^{n-1}\right)
\end{aligned}
$$


With this rearrangement we can calculate $\psi_{k}^{n}$ from $\psi_{k}^{n-1}$. There is no need to save the total history of $v(t)$ which results in considerable memory savings. The derivatives in Eqs. (B5) and (1) are discretized, accurate to 2nd order (Taflove, 1998) using central finite differences both in space and in time. We obtain

$$
\begin{aligned}
\frac{i_{k+1}^{n}-i_{k-1}^{n}}{2 \Delta x}= & -G_{k}^{\prime} v_{k}^{n}-C_{0 k}^{\prime} \epsilon_{\infty k}^{\prime} \frac{v_{k}^{n+1}-v_{k}^{n-1}}{2 \Delta t} \\
& -C_{0 k}^{\prime} \frac{\Delta \epsilon_{k}^{\prime}}{\tau_{k}} v_{k}^{n}+C_{0 k}^{\prime} \frac{\Delta \epsilon_{k}^{\prime}}{\tau_{k}^{2}} \psi_{k}^{n}
\end{aligned}
$$

$\frac{v_{n+1}^{n}-v_{k-1}^{n}}{2 \Delta x}=-R_{k}^{\prime} i_{k}^{n}-L_{k}^{\prime} \frac{i_{k}^{n+1}-i_{k}^{n-1}}{2 \Delta t}$.

By rearranging terms this leads to the update procedure for voltage and current

$$
\begin{aligned}
v_{k}^{n+1}= & -\frac{2 \Delta t G_{k}^{\prime}}{C_{0 k}^{\prime} \epsilon_{\infty k}^{\prime}} v_{k}^{n}-\frac{2 \Delta t \Delta \epsilon_{k}^{\prime}}{\epsilon_{\infty k}^{\prime} \tau_{k}} v_{k}^{n}+v_{k}^{n-1} \\
& -\frac{\Delta t}{C_{0 k}^{\prime} \epsilon_{\infty k}^{\prime} \Delta x}\left(i_{k+1}^{n}-i_{k-1}^{n}\right)+\frac{2 \Delta \epsilon_{k}^{\prime} \Delta t}{\tau_{k}^{2} \epsilon_{\infty k}^{\prime}} \psi_{k}^{n}
\end{aligned}
$$

$$
i_{k}^{n+1}=-\frac{2 R_{k}^{\prime} \Delta t}{L_{k}^{\prime}} i_{k}^{n}+i_{k}^{n-1}-\frac{\Delta t}{\Delta x L_{k}^{\prime}}\left(v_{k+1}^{n}-v_{k-1}^{n}\right) .
$$

\section{Appendix C}

\section{List of symbols}

$\begin{array}{ll}\boldsymbol{B} & \text { magnetic field, } \frac{\mathrm{Vs}}{\mathrm{m}^{2}}=\mathrm{T} . \\ c_{0} & \text { speed of light } \mathrm{m} \text { vacuum, } \frac{\mathrm{m}}{\mathrm{s}} . \\ C & \text { capacitance, } \mathrm{F} . \\ C_{T} & \text { value of the capacitor terminating } \\ & \text { the TDR probe, } \mathrm{F} . \\ C^{\prime} & \text { capacitance per unit length of a } \\ & \text { transmission line, } \frac{\mathrm{F}}{\mathrm{m}} . \\ \delta(t) & \text { Dirac delta function. } \\ \Delta x & \text { spatial resolution in the discretiza- } \\ & \text { tion of the transmission line equa- } \\ & \text { tions, m. } \\ & \text { difference between static permittiv- } \\ & \text { ity and permittivity at infinite fre- } \\ & \text { quency, dimensionless. }\end{array}$

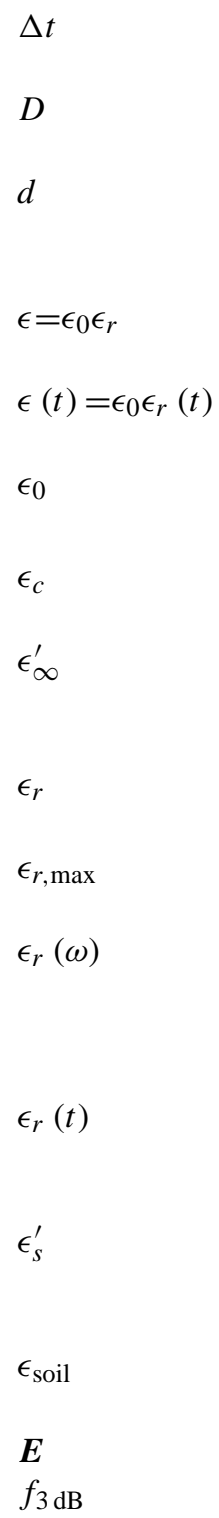

$f_{\max }$

$f_{\text {rel }}$

$G$

$G^{\prime}$

I

$I_{T}$

$i(x, t)$

$i_{k}^{n} \equiv\left(x_{k}, t_{n}\right)$

$i_{S}$

$j$

$j$ discretization width in the time domain, $\mathrm{s}$.

diameter of the conductors of a twoor three-wire transmission line, $\mathrm{m}$. distance between the centers of two nearest conductors of a transmission line, $\mathrm{m}$.

absolute complex dielectric permittivity, $\frac{\mathrm{As}}{\mathrm{Vm}}$.

absolute dielectric permittivity as function of time, $\frac{\mathrm{As}}{\mathrm{Vm}}$.

absolute dielectric permittivity of vacuum, $\frac{1}{\mu \mathrm{c}^{2}}$.

effective relative permittivity of a composite medium, dimensionless. real valued relative permittivity at infinite frequency in Debye model, dimensionless.

complex valued relative dielectric permittivity, dimensionless.

maximum value of relative dielectric permittivity, dimensionless.

complex valued relative dielectric permittivity as a function of angular frequency of electric field, dimensionless.

relative dielectric permittivity as a function of time, Fourier transformed of $\epsilon_{r}(\omega)$, dimensionless.

real valued relative permittivity at zero frequency in Debye model, dimensionless.

relative permittivity or a soil matrix without water, dimensionless.

electric field, $\frac{\mathrm{v}}{\mathrm{m}}$.

frequency at which amplitude of the respective function has reduced by $3 \mathrm{~dB}, \mathrm{~Hz}$.

maximum frequency, $\mathrm{Hz}$.

relaxation frequency in Debye model, $\mathrm{Hz}$.

conductance, $\mathrm{S}$.

conductance per unit length of a transmission line, $\frac{\mathrm{S}}{\mathrm{m}}$.

current, A.

current at the end of the transmission line, A.

current on a transmission line as function of position $x$ and time $t$, A. current at point $k \Delta x$ at time $n \Delta t$, A.

TDR source current, A. imaginary unit, $j=\sqrt{-1}$. current density, $\frac{\mathrm{A}}{\mathrm{m}^{2}}$. 


\begin{tabular}{|c|c|c|}
\hline$j_{x}, j_{y}, j_{z}$ & $\begin{array}{l}\text { components of current density re- } \\
\text { ferring to a Cartesian coordination } \\
\text { system, } \mathrm{A} \text {. }\end{array}$ & $\begin{array}{l}R_{\text {skin }} \\
R_{T}\end{array}$ \\
\hline$\kappa=\frac{d}{D}$ & $\begin{array}{l}\text { factor of probe geometry, dimen- } \\
\text { sionless. }\end{array}$ & $\sigma(x)$ \\
\hline$k$ & $\begin{array}{l}\text { index used for the specification of } \\
\text { spatial locations, } k \cdot \Delta x=x_{k} \text {, dimen- } \\
\text { sionless. }\end{array}$ & $\tau=\frac{1}{2 \pi f_{\mathrm{rel}}}$ \\
\hline$K$ & $\begin{array}{l}\text { index, denoting the last index in } \\
\text { spatial discretization, } K \cdot \Delta x=\Lambda \text {, } \\
\text { dimensionless. }\end{array}$ & $\begin{array}{l}\theta \\
\theta(x)\end{array}$ \\
\hline$\Lambda$ & total length of TDR-probe, $\mathrm{m}$. & \\
\hline$\lambda_{\min }$ & minimum wavelength, $\mathrm{m}$. & \\
\hline$L^{\prime}$ & $\begin{array}{l}\text { inductance per unit length of a } \\
\text { transmission line, } \frac{\mathrm{H}}{\mathrm{m}} \text {. }\end{array}$ & $t_{\text {rise }}$ \\
\hline$l^{l}=\mu_{0} \mu_{r}$ & $\begin{array}{l}\text { length of a part of TDR probe, } \mathrm{m} \text {. } \\
\text { magnetic permeability of a mate- } \\
\text { rial, } \frac{\mathrm{Vs}}{\mathrm{Am}} \text {. }\end{array}$ & \\
\hline$\mu_{0}$ & $\begin{array}{l}\text { magnetic permeability of vacuum, } \\
4 \pi 10^{-7}, \frac{\mathrm{Vs}}{\mathrm{Am}}\end{array}$ & $t_{\mathrm{sec}}$ \\
\hline$\mu_{r}=\left(\mu_{r}^{\prime}-j \mu_{r}^{\prime \prime}\right)$ & $\begin{array}{l}\text { complex valued relative magnetic } \\
\text { permeability, equals } 1 \text { for consid- } \\
\text { ered soil materials, dimensionless. }\end{array}$ & $\begin{array}{l}U \\
V \\
V_{T}\end{array}$ \\
\hline$\mu_{r}^{\prime}$ & $\begin{array}{l}\text { real part of the complex valued rel- } \\
\text { ative magnetic permeability, dimen- } \\
\text { sionless. }\end{array}$ & $v(x, t)$ \\
\hline$\mu_{r}^{\prime \prime}$ & $\begin{array}{l}\text { imaginary part of the complex val- } \\
\text { ued relative magnetic permeability, } \\
\text { dimensionless. } \\
\text { number of fitness envelope points }\end{array}$ & $\begin{array}{l}v_{S} \\
x, y, z\end{array}$ \\
\hline
\end{tabular}

skin resistance of a conductor, $\Omega$. value of the resistor terminating the TDR probe, $\Omega$.

ohmic conductivity as a function of longitudinal position on the TDR probe, $\frac{\mathrm{S}}{\mathrm{m}}$.

relaxation time of a dipole in the Debye model, s.

volumetric water content, $\frac{\mathrm{m}^{3}}{\mathrm{~m}^{3}}$.

volumetric water content as function of longitudinal position on the TDR probe, $\frac{\mathrm{m}^{3}}{\mathrm{~m}^{3}}$.

time, $s$.

rise time of an electrical signal, usually the time required for the signal to rise from 10 to $90 \%$ of its final value, $s$.

time step security for explicit time domain integration, $\mathrm{s}$.

Heaviside step function.

voltage, $\mathrm{V}$.

voltage at the end of the transmission line, $\mathrm{V}$.

voltage on a transmission line as function of position $x$ and time $t, \mathrm{~V}$. voltage at point $k \Delta x$ at time $n \Delta t$, $\mathrm{V}$.

TDR source voltage, $\mathrm{V}$. spatial coordinate, $\mathrm{m}$.

Acknowledgements. We greatly appreciate the critical and constructive comments of C. Hübner and S. Schlaeger which helped us to qualitatively improve the manuscript.

We are most grateful to M. Laudien of Ansoft Inc. whose generous support with an $\mathrm{HFSS}^{\mathrm{TM}}$ license was essential for validating the analytical transmission line model and for benchmarking our TDR code.

Financial support of this work was provided in part by Deutsche Forschungsgemeinschaft (Project No. 1080-8/1\&2).

Edited by: E. Zehe

time, $x(n \cdot \Delta t)=x^{n}$, dimensionless.

angular frequency of electric field, $\frac{1}{\mathrm{~s}}$.

electro static potential, $\mathrm{V}$.

$=e^{-\frac{\Delta t}{\tau_{k}}} \psi_{k}^{n-1}+\frac{\Delta t}{2}\left(v_{k}^{n}+e^{-\frac{\Delta t}{\tau_{k}}} v_{k}^{n-1}\right)$

abbreviation for calculations in a dispersive dielectric medium.

electric charge, As.

reflection coefficient, dimensionless.

resistance per unit length of a transmission line, $\frac{\Omega}{\mathrm{m}}$.

$R_{S} \quad$ source impedance of resistive voltage source, $\Omega$.

\section{References}

Birchak, J. R., Gardner, C. G., Hipp, J. E., and Victor, J. M.: High Dielectric Constant Microwave Probes for Sensing Soil Moisture, Proceedings of the IEEE, 62, 93-98, 1974.

Dasberg, S. and Dalton, F. N.: Time Domain Reflectometry Field Measurements of Soil Water Content and Electrical Conductivity, Soil Sci. Soc. Am. J., 49, 293-297, 1985.

Dasberg, S. and Hopmans, J. W.: Time Domain Reflectometry Calibration for Uniformly and Nonuniformly Wetted Sand and Clayed Loam Soils, Soil Sci. Soc. Amer. J., 56, 1341-1345, 1992. 
Debye, P.: Polare Molekeln, Verlag von S. Hirzel, Leipzig, 1929.

Feng, W., Lin, C. P., Deschamps, R. J., and Drnevich, V. P.: Theoretical model of a multisection time domain reflectometry measurement system, Water Resour. Res., 35, 2321-2331, 1999.

Heimovaara, T. J.: Frequency domain analysis of time domain reflectometry waveforms, 1 . Measurement of the complex dielectric permittivity of soils, Water Resour. Res., 2, 189-200, 1994.

Heimovaara, T. J., de Winter, E. J. G., van Loon, W. K. P., and Esveld, D. C.: Frequency-dependent dielectric permittivity from 0 to $1 \mathrm{GHz}$ : Time domain reflectometry measurements compared with frequency domain network analyzer measurements, Water Resour. Res., 32, 3603-3610, 1996.

Heimovaara, T. J., Huisman, J. A., Vrugt, J. A., and Bouten, W.: Obtaining the Spatial Distribution of Water Content along a TDR probe Using the SCEM-UA Bayesian Inverse Modeling Scheme, Vadose Zone J., 3, 1128-1145, 2004.

Hilhorst, M. A., Dirksen, C., Kampers, F. W. H., and Feddes, R. A.: Dielectric Relaxation of Bound Water versus Soil Matric Pressure, Soil Sci. Soc. Amer. J., 65, 311-314, 2001.

Hoekstra, P. and Delaney, A.: Dielectric Properties of Soils at UHF and Microwave Frequencies, J. Geophys. Res., 79, 1699-1708, 1974.

Hook, W. R., Livingston, N. J., Sun, Z. J., and Hook, P. B.: Remote Diode Shorting Improves Measurement of Soil Water by Time Domain Reflectometry, Soil Sci. Soc. Amer. J., 56, 1384-1391, 1992.

Huisman, J. A., Bouten, W., and Vrugt, J. A.: Accuracy of frequency domain analysis scenarios for the determination of complex dielectric permittivity, Water Resour. Res., 40, 1-12, 2004.

Ishida, T., Makino, T., and Wang, C.: Dielectric-relaxation spectroscopy of kaolinite, montmorillonite, allophane, and imogolite under moist conditions, Clays Clay Miner., 48, 75-84, 2000.

Kunz, K. S. and Luebbers, R. J.: The Finite Difference Time Domain Method for Electromagnetics, CRC Press, 2000 Corporate Blvd., N. W., Boca Raton, Florida, 1993.

Levine, D.: Users Guide to the PGAPack Parallel Genetic Algorithm Library, Tech. rep., Argonne National Laboratory 95/18, 9700 South Cass Avenue, Argonne Il 60439, 1996.

Lin, C. P.: Analysis of non-uniform and dispersive time domain reflectometry measurement systems with application to the dielectric spectroscopy of soils, Water Resour. Res., 39, 2003.

Lundstedt, J. and Ström, S.: Simultaneous reconstruction of two parameters from the transient response of a nonuniform LCRG transmission line, J. Electromagnet. Wave., 10, 19-50, 1996.

Norgren, M. and He, S.: An optimization approach to the frequency-domain inverse problem far a nonuniform LCRG transmission line, IEEE T. Microw. Theory, 44, 8, 1503-1507, 1996.

Nussberger, M., Benedickter, H. R., Bächtold, W., Flühler, H., and Wunderli, H.: Single-Rod Probes for Time Domain Reflectometry: Sensitivity and Calibration, Vadose Zone J., 4, 551-557, doi:10.2136/vzj2004.0093, 2005.

Nyfors, E. and Vainikainen, P.: Industrial Microwave Sensors, Artech House, INC., 685 Canton Street, Norwood, MA 02062, USA, 1989.

Oswald, B.: Full Wave Solution of Inverse Electromagnetic Problems - Applications in Environmental Measurement Techniques, Ph.D. thesis, Swiss Federal Institute of Technology, Zurich, 2000.
Oswald, B., Benedickter, H. R., Bächtold, W., and Flühler, H.: Spatially resolved water content profiles from inverted TDR signals, Water Resour. Res., 39, doi:10.1029/2002WR001890, 2003.

Oswald, B., Benedickter, H. R., Bächtold, W., and Flühler, H.: A single rod probe for time domain reflectometry, Vadose Zone J., 3, 1152-1159, 2004.

Pereira, D. S.: Développement d'une nouvelle méthode de détermination des profils de teneur en eau dans les sols par inversion d'un signal TDR, Ph.D. thesis, Lab. d'Etude des Transf. en Hydrol. et Environ (LTHE), Univ. Joseph Fourier-Grenoble I, Grenoble, France, 1997.

Rahmat-Samii, Y. and Michielssen, E.: Electromagnetic Optimization by Genetic Algorithms, Wiley Series in Microwave and Optical Engineering, John Wiley \& Sons, 1999.

Ramo, S., Whinnery, J. R., and van Duzer, T. V.: Fields and Waves in Communication Electronics, John Wiley \& Sons, New York, 2 edn., 1984.

Robinson, D. A., Jones, S. B., Wraith, J. M., Or, D., and Friedman, S. P.: A Review of Advances in Dielectric and Electrical Conductivity Measurements in Soils Using Time Domain Reflectometry, Vadose Zone J., 2, 444-475, 2003.

Robinson, D. A., Schaap, M. G., Or, D., and Jones, S. B.: On the effective measurement frequency of time domain reflectometry in dispersive and non-conductive dielectric materials, Water Resour. Res., 41, 2005.

Roth, K., Schulin, R., Flühler, H., and Attinger, W.: Calibration of Time Domain Reflectometry for Water Content Measurement Using a Composite Dielectric Approach, Water Resour. Res., 26, 2267-2273, 1990.

Schlaeger, S.: A fast TDR-inversion technique for the reconstruction of spatial soil moisture content, Hydrol. Earth Syst. Sci., 9, 481-492, 2005.

Sposito, G. and Prost, R.: Structure of Water Adsorbed on Smectites, Chemical Reviews, 82, 553-573, 1982.

Taflove, A.: Computational electrodynamics: the finite difference time domain method, Artech House, Norwood, Massachusetts, 1998.

Todoroff, P., Lorion, R., and Lan Sun Luk, J. D.: L'utilisation des algorithmes génétiques pour l'identification de profil hydriques de sol a partir de courbes réflectrométriques, C. R. Acad. Sci. Ser. IIa, Sci. Terre Planetes, 327, 607-610, 1998.

Topp, G. C. and Davis, J. L.: Measurement of Soil Water Content using Time-domain Reflectometry (TDR): A Field Evaluation, Soil Sci. Soc. Amer. J., 49, 19-24, 1985.

Topp, G. C., Davis, J. L., and Annan, A. P.: Electromagnetic Determination of Soil Water Content: Measurement in Coaxial Transmission Lines, Water Resour. Res., 16, 574-582, 1980.

Topp, G. C., Davis, J. L., and Annan, A. P.: Electromagnetic Determination of Soil Water Content Using TDR: I. Applications to Wetting Fronts and Steep Gradients, Soil Sci. Soc. Amer. J., 46, 672-678, 1982a.

Topp, G. C., Davis, J. L., and Annan, A. P.: Electromagnetic Determination of Soil Water Content Using TDR: II. Evaluation of Installation and Configuration of Parallel Transmission Lines, Soil Sci. Soc. Amer. J., 46, 678-684, 1982 b.

Volakis, J. L., 'Chatterjee, A., and Kempel, L. C.: The Finite Element Method for Electromagnetics, IEEE Press, New York, 1998. 
Wollschläger, U. and Roth, K.: Estimation of Temporal Changes of Volumetric Soil Water Content from Ground-Penetrating Radar Reflections, Subsurface Sensing Technol. Appl., 6, 201-218, 2005 .
Yanuka, M., Topp, G. C., Zegelin, S., and Zebchuk, W. D.: Multiple Reflection and Attenuation of Time Domain Reflectometry Pulses: Theoretical Considerations for Applications to Soil and Water, Water Resour. Res., 24, 939-944, 1988. 